\title{
Hypothalamic miR-103 Protects from Hyperphagic Obesity in Mice
}

\author{
ํㅣㄱya A. Vinnikov, ${ }^{1}$ Karolina Hajdukiewicz, ${ }^{3}$ Jürgen Reymann, ${ }^{5}$ Jürgen Beneke, ${ }^{5}$ Rafal Czajkowski, ${ }^{3}$ Lena C. Roth, ${ }^{2,4,7}$ \\ Martin Novak, ${ }^{1}$ Andreas Roller, ${ }^{8}$ Nicole Dörner, ${ }^{1}$ Vytaute Starkuviene, ${ }^{6}{ }^{\circ}$ Fabian J. Theis, ${ }^{8,9}$ Holger Erfle, ${ }^{5}$ \\ Günther Schütz, ${ }^{1}$ Valery Grinevich, ${ }^{2,4,7 *}$ and Witold Konopka ${ }^{1,3 *}$ \\ ${ }^{1}$ Molecular Biology of the Cell I, ${ }^{2}$ Schaller Research Group on Neuropeptides, German Cancer Research Center, 69120 Heidelberg, Germany, ${ }^{3}$ Laboratory of \\ Animal Models, Neurobiology Center, Nencki Institute of Experimental Biology of the Polish Academy of Sciences, 02-093 Warsaw, Poland, ${ }^{4}$ CellNetworks \\ Cluster of Excellence, ${ }^{5}$ ViroQuant-CellNetworks RNAi Screening Facility, BioQuant and ${ }^{6}$ ViroQuant Screening of Cellular Networks, BioQuant, University of \\ Heidelberg, 69120 Heidelberg, Germany, ${ }^{7}$ Department of Molecular Neurobiology, Max Planck Institute for Medical Research, 69120 Heidelberg, Germany, \\ ${ }^{8}$ Institute for Bioinformatics and Systems Biology, Helmholtz Center Munich, German Research Center for Environmental Health, 85764 Neuherberg, \\ Germany, and ${ }^{9}$ Department of Mathematics, Technical University Munich, 85748 Garching, Germany
}

The role of neuronal noncoding RNAs in energy control of the body is not fully understood. The arcuate nucleus (ARC) of the hypothalamus comprises neurons regulating food intake and body weight. Here we show that Dicer-dependent loss of microRNAs in these neurons of adult (DicerCKO) mice causes chronic overactivation of the signaling pathways involving phosphatidylinositol-3-kinase (PI3K), Akt, and mammalian target of rapamycin (mTOR) and an imbalance in the levels of neuropeptides, resulting in severe hyperphagic obesity. Similarly, the activation of PI3K-Akt-mTOR pathway due to Pten deletion in the adult forebrain leads to comparable weight increase. Conversely, the mTORC1 inhibitor rapamycin normalizes obesity in mice with an inactivated Dicer1 or Pten gene. Importantly, the continuous delivery of oligonucleotides mimicking microRNAs, which are predicted to target PI3K-Akt-mTOR pathway components, to the hypothalamus attenuates adiposity in DicerCKO mice. Furthermore, loss of miR-103 causes strong upregulation of the PI3K-Akt-mTOR pathway in vitro and its application into the ARC of the Dicer-deficient mice both reverses upregulation of Pik3cg, the mRNA encoding the catalytic subunit p $110 \gamma$ of the PI3K complex, and attenuates the hyperphagic obesity. Our data demonstrate in vivo the crucial role of neuronal microRNAs in the control of energy homeostasis.

Key words: Dicer; hypothalamus; metabolism; mice; microRNA; obesity

\section{Introduction}

Obesity affects $>10 \%$ of people all over the world with the number of cases increasing at an especially high rate in developed countries (Finucane et al., 2011). Obesity, characterized by an

\section{Received Oct. 4, 2013; revised June 26, 2014; accepted July 2, 2014.}

Author contributions: I.A.V., F.J.T., G.S., V.G., and W.K. designed research; I.A.V., K.H., J.B., L.C.R., N.D., H.E., and W.K. performed research; V.S. and H.E. contributed unpublished reagents/analytic tools; I.A.V., J.R., R.C., M.N., A.R., F.J.T., H.E., and W.K. analyzed data; I.A.V., A.R., V.G., and W.K. wrote the paper.

This work was supported by the Deutsche Forschungsgemeinschaft (DFG) through Grant SFB488, the European Union through Grant LSHM-CT-2005-018652 (CRESCENDO), the German Federal Ministry of Education and Research through German Genome Research Network-Plus Grants FZK-01GS08153 and 01GS08142, and the Hermann von Helmholtz Gemeinschaft Deutscher Forschungszentren through Initiative CoReNe (Network II, E2) to G.S.; the Helmholtz Alliance on Systems Biology (CoReNe), the European Research Council (Starting Grant: Latent Causes), and the DFG Schwerpunktprogramm on Informations- und Kommunikationstheorie in der Molekularbiologie to F.T.; the Max Planck Society, DFG Grants GR-3619/2-1, 3619/3-1, 3619/4-1, and the Chica and Heinz Schaller Research Foundation to V.G.; and the National Science Centre (Poland) Grant (SONATA) 2011/01/D/NZ4/03744 to W.K. We thank Mathias Merkenschlager for providing Dicer ${ }^{\mathrm{f} / \mathrm{f}}$ mice; Heike Alter, Nina Beil, Slawek Mazur, Andrii Domanskyi, Sergey Khrulev, Tabea Arnsperger, and Annette Kopp-Schneider for technical help; and Anne Seller for proofreading the manuscript.

The authors declare no competing financial interests.

*V.G. and W.K. contributed equally to this work.

Correspondence should be addressed to any of the following: llya Vinnikov at the above address. E-mail: ilya.vinnikov@gmail.com; or Valery Grinevich at the above address. E-mail: valery.grinevich@mpimf-heidelberg.mpg.de; or Witold Konopka, Pasteur 3,02-093 Warsaw, Poland. E-mail: w.konopka@nencki.gov.pl.

DOI:10.1523/JNEUROSCI.4251-13.2014

Copyright $\odot 2014$ the authors $\quad 0270-6474 / 14 / 3410659-16 \$ 15.00 / 0$ excess of fat content in the body, is the major risk factor for cardiovascular diseases, type 2 diabetes mellitus, and some cancers and it is the world's fifth leading risk for death. Control of food intake or energy accumulation and expenditure is achieved by both peripheral tissues and the CNS. The brain nuclei most important for energy homeostasis are primarily located in the hypothalamus (Elmquist et al., 1999; Myers et al., 2009).

The first order metabolic sensors in this region are foodintake stimulating neurons expressing agouti-related protein and neuropeptide Y (AgRP/NPY) and anorexigenic proopiomelanocortin (POMC)-expressing neurons (Xu et al., 2011). Both types of cells are located within the arcuate nucleus (ARC) of the hypothalamus. Plasma hormones in concert with nutrients transmit a message about energy status to these neurons (Belgardt and Brüning, 2010). This information affects a variety of intracellular signal transduction pathways, including the phosphatidylinositol-3-kinase (PI3K)-Akt-mammalian target of rapamycin (mTOR) pathway, modulating metabolic responses in the peripheral tissues (Friedman and Halaas, 1998; Seeley and Woods, 2003; Ryan et al., 2012). Transcriptional, translational, and post-translational control of components of these pathways in the ARC neurons contributes to CNS-controlled energy homeostasis. 
MicroRNAs guide the silencing machinery to the target mRNA to suppress its translation and/or stability in various cell types, including neurons (Bartel, 2009; Schratt, 2009; Mikl et al., 2010). Previously we have demonstrated that the loss of Dicer, a key nuclease for microRNA maturation, modifies neuronal activity in the hippocampus (Konopka et al., 2010). With regard to obesity, an upregulation of specific microRNAs resulting in adverse effects on glucose metabolism via the PI3K-Akt-mTOR pathway has been shown in liver or adipose tissue (Jordan et al., 2011; Trajkovski et al., 2011; Kornfeld et al., 2013). However, contribution of neuronal microRNAs in the central control of metabolism is poorly studied.

\section{Materials and Methods}

Animals. All animal experimental procedures were conducted on the C57BL/6N genetic background ( $\geq 9$ backcrosses) at the German Cancer Research Center in accordance with institutional and international standards and approved by the local authorities (Regierungspräsidium) of the state of Baden-Württemberg. Mice of both sexes were used for all experiments, unless otherwise stated. Mice with disrupted Dicer1 or Pten genes in forebrain neurons Dicer1 CaMKCreERT2 (Cobb et al., 2005; Erdmann et al., 2007) or Pten CaMKCreERT2 (Lesche et al., 2002; named in the text and figures as DicerCKO or PtenCKO, respectively) were used. As a control, we used either littermates negative for the CreERT2 transgene, $\operatorname{Dicer}^{\mathrm{f} / \mathrm{fl}}$ or Pten ${ }^{\mathrm{f} / \mathrm{fl}}$, or heterozygous Dicer ${ }^{\mathrm{wt} / \mathrm{fl}}$ mice positive for the CreERT2 because they did not differ with regard to the observed phenotype. Inactivation of the Dicer 1 or Pten genes was obtained by injection of $1 \mathrm{mg}$ of tamoxifen (TAM) twice per day for 5 consecutive days in 8-10-weekold animals, with the exception of the DicerCKO ${ }^{4 \times T A M}$ and RosaLacZ CaMKCreERT2-4×TAM groups, which differ from the other animals only by the regimen of TAM treatment ( $1 \mathrm{mg}$ twice per day for 2 consecutive days). Approximately $90 \%$ of DicerCKO ${ }^{4 \times \mathrm{TAM}}$ mice developed the hyperphagic obesity phenotype and only those were used for analyses. Animals were maintained on a $12 \mathrm{~h}$ light/dark cycle with ad libitum access to water and food (standard chow food \#3437, Kliba Nafag). Measurements of food intake and weight were done weekly starting from week 1 following TAM treatment (post-TAM). Phenotypic analyses were performed at the peak of the obesity phenotype, i.e., week 6 after the recombination event, unless otherwise stated. The mice were killed during the light phase in a fed state. For a pair-fed experiment, DicerCKO animals were given each day the same amount of food $(\sim 4 \mathrm{~g})$ that control individuals consume daily on average. In both genders, some of the PtenCKO mice failed to manifest the weight increase ( $n=3$ and 7 of 9 for females and males, respectively) and were excluded from the analysis. Longacting basal insulin analog (subcutaneous) and rapamycin (intraperitoneal) were applied daily from from the 2 nd to the 8 th week after the mutation onset at dose $1.25 \mathrm{IU}$ and $12 \mathrm{mg} / \mathrm{kg}$ body weight, respectively. Reporter lines for Cre-dependent recombination in the CaMKCreERT2 mouse line were as follows: Rosa26-tdTomato, Rosa26-LacZ (Soriano, 1999; Madisen et al., 2010). For the quantification of the POMC neurons, the 12-week-post-TAM DicerCKO or heterozygous littermates on the background of the FVB-Tg(Pomcl-hrGFP)1Lowl/J [Research Resource Identifier (RRID): IMSR_JAX:006421, referred as POMC-GFP] transgenic line (Jackson Laboratory) were used $(n=2)$. Briefly, the mice were perfused, the brains were vibratome-sliced (thickness, $40 \mu \mathrm{m}$ ), and the number of GFP-positive neurons per section was blindly quantified in each of the 16 sequential sections per mouse. The sum of the neurons per mouse was used for subsequent analyses.

$X$-ray densitometry and fat pad analyses. Measurements of the body composition were performed on the xylazine-ketamine anesthetized animals by the automated mouse $\mathrm{x}$-ray densitometer system PIXI-mus II (Lunar). The outlined red area containing skull and metal earmarks (see Fig. $2 B$, arrows) was excluded from the measurements according to the manufacturer protocol.

TaqMan analysis and transcriptome array profiling. For NPY, AgRP, and POMC mRNA detection in the hypothalamus of control and DicerCKO animals, TaqMan probes Mm00445771_m1, Mm00475829_m1, and Mm00435874_m1 (Applied Biosystems) were used, respectively. For the isolation of ARC, the brain of 6-week-post-TAM DicerCKO or control mice injected with miR-103-mimic or scrambled oligonucleotides was sliced in the brain matrix immediately after the optical chiasm and $2 \mathrm{~mm}$ caudally from the first cut. ARC was microdissected from the resulting slice by two scalpels and the total RNA was obtained by the RNeasy Mikro kit (Qiagen), according to the manufacturer's protocol. The Taqman probes for Pik3cg, Pik3rl, and Hprt mRNA were as follows: Mm00445038_m1, Mm00803160_m1, and Mm01545399_m1, respectively. TaqMan RT-PCRs were performed according to the manufacturer's protocol (Applied Biosystems). MicroRNA profiling (518 microRNAs in total) was performed using the TaqMan MicroRNA Array version 2.0 method on the hypothalamus-isolated RNA (mirVana microRNA Isolation kit, Ambion) of C57BL/6 mice according to the protocol of the manufacturer. The Mouse Sentrix- 6 array mRNA profiling (Illumina) was performed according to the manufacturer's protocol.

Histological analyses, ELISA, and immunoblotting. For immunohistochemical analysis, mice were transcardially perfused with $4 \%$ paraformaldehyde and brains were postfixed at $4^{\circ} \mathrm{C}$ for $24 \mathrm{~h}$. Coronal vibratome sections $(50 \mu \mathrm{m})$ were processed for immunohistochemistry using the Vectastain $\mathrm{ABC}$ system (Vector Laboratories) and diaminobenzidine DAB (Sigma-Aldrich) staining. The following primary antibodies were used: phospho-S6 ribosomal protein (S235/236) Rb mAb (1:100; catalog \#4857S; RRID: AB_2181035, Cell Signaling Technology) and anti-Cre antibody (1:3000; Kellendonk et al., 1999). Quantification of the pS6RPpositive and Cre-positive cells was performed by MCID Image Analysis software (InterFocus Imaging) in $\geq 12$ sections per animal. Other antibodies used for immunohistochemical and immunofluorescent analyses were as follows: ACTH (1:1000; Parlow; 1:50; National Institutes of Health Pituitary Program), NPY (Phoenix Pharmaceuticals), anti-fluorescein-Alexa Fluor 594 conjugate (catalog \#A11091; RRID: AB_1500116, Invitrogen). For $\beta$-galactosidase staining, animals were perfused with $4 \%$ paraformaldehyde and brains were dissected and postfixed for $2 \mathrm{~h}$. Next, brains were cut on vibratome into $100-\mu \mathrm{m}$-thick sections. The $\mathrm{x}$-gal staining procedure was performed as described earlier (Erdmann et al., 2007). Next, the sections were counterstained with nuclear fast red (Sigma-Aldrich) and mounted with Eukitt (O. Kindler). Fluorescent signals in the brain samples were visualized directly with confocal system TSC SP5 (Leica) or LSM 780 (Zeiss). The latter was also used for linear unmixing for direct detection of the FAM (amine-reactive succinimidyl esters of carboxyfluorescein) signal. TUNEL assay (Roche) was performed as described earlier (Isermann et al., 2007). To detect protein levels in the hypothalamus of DicerCKO and control mice by a standard Western blot analysis, the following antibodies were used: signal transducer and activator of transcription 3 (Stat3) rabbit Ab (1:1000; Cell Signaling Technology) and pStat3 (Y705; D3A7) rabbit mAb (1:1000; catalog \#9145S; RRID: AB_561305, Cell Signaling Technology). Images were acquired with the LAS-3000 imaging system (Fuji); band intensity was quantified using ImageGauge software (Fuji) and normalized to that of control samples. Perigonadal and perirenal fat tissue was dissected, weighed, postfixed in $4 \%$ paraformaldehyde at $4^{\circ} \mathrm{C}$ for $24 \mathrm{~h}$, and embedded in paraffin for hematoxylin and eosin staining.

Leptin and insulin concentrations were determined by ELISA kit (Crystal Chem) in the plasma supplied with EDTA according to the manufacturer's protocol. The hypothalmi were homogenized in the solution of $1 \% \mathrm{SDS}, 10 \mathrm{~mm} \mathrm{NaF}$, and $10 \mathrm{~mm} \mathrm{Na}_{3} \mathrm{VO}_{4}$. The protein concentration of the extracts was determined and the same amount of proteins for each sample was measured by NPY, $\alpha$-MSH, or AgRP ELISA (Phoenix Pharmaceuticals), according to the manufacturer's protocol.

Virus-mediated Dicerl gene deletion. Production and purification of recombinant adeno-associated viral (AAV) vectors (Serotype 1/2), equipped with the chicken $\beta$-actin promoter $(1 \mathrm{~kb})$ driving humanized version of Cre, were as described previously (Knobloch et al., 2012). The genomic titers of the viral vectors $\left[\sim 10^{10}\right.$ genomic copies per microliter for recombinant AAV vector-Cre (rAAV-Cre)] were determined with QuickTiter AAV Quantitation Kit (Cell Biolabs) and RT-PCR using the ABI 7700 cycler (Applied Biosystems). The surgery and bilateral stereotaxic injections of Dicer ${ }^{\mathrm{f} / \mathrm{fl}}$ mice were performed as described previously (Knobloch et al., 2012). In one experiment, animals were given two 
injections at indicated stereotaxic positions (Table 1) with $0.14 \mu \mathrm{l}$ per injection site (see Fig. 2D). This huge amount of viral particles did not allow us to restrict the infection only to the regions of choice so that we always had a large number of cells recombined above the target regions. In the other experiment, we induced the Dicerl gene recombination, adjusting it to the shape of the ARC: mice were given 6 or 10 injections with $0.035 \mu \mathrm{l}$ or $0.01 \mu \mathrm{l}$ per injection site (see Fig. $2 E$ ), respectively. This allowed us to reduce drastically the amount of rAAV-Cre per injection site and thus to minimize a possible influence from other hypothalamic structures. Animals injected with mock viral vector into ARC or with Dicer deletion in dorsomedial hypothalamic nucleus (DMH), paraventricular nucleus (PVN), lateral hypothalamus ( $\mathrm{LH})$, or the basolateral amygdala (BLA) served as control groups. The stereotaxic coordinates (Paxinos and Franklin, 2001) and numbers of mice are presented in Table 1. The animals that did not show recombination in ARC, as shown by Cre staining (see Fig. 2E), were excluded from analysis. Importantly, ARC-injected animals revealed no signs of destruction of the nucleus that may potentially lead to the obesity phenotype (Xu et al., 2011).

MicroRNA target prediction. Various microRNA target prediction tools have been developed in the past using different approaches. Due to imperfect base pairing and the short length of microRNA binding sites, the prediction often yields false-positive target genes. It has been shown previously that the combination of the different prediction tools can yield an improved specificity with only a marginal decrease in sensitivity relative to any individual algorithm (Sethupathy et al., 2006). To address this important issue, we applied the miTALOS Web resource, which combines several distinct microRNA target prediction tools based on sequence similarity, conservation, site accessibility, or pattern-based approaches (Kowarsch et al., 2011). MiTALOS is able to combine $\leq 5$ different microRNA target prediction tools, such as TargetScanS, RNA22, PicTar, PiTa, and TargetSpy. Moreover, it identifies potential microRNA-pathway associations by an enrichment analysis of microRNA target genes. Pathway information was integrated from the Kyoto Encyclopedia of Genes and Genomes (KEGG; Kanehisa et al., 2008). The enrichment approach identifies microRNA-pathway associations using the number of target genes in a specific pathway (Kowarsch et al., 2011). For the identification of potential associations between microRNAs and the PI3K-Akt-mTOR pathway, we predicted associations only for microRNAs being highly abundant in the hypothalamus (www. dkfz.de/de/molekularbiologie/download/Vinnikov-et-al-S.xlsx) using all five different microRNA target prediction tools provided by miTALOS (www.dkfz.de/de/molekularbiologie/download/Vinnikov-et-al-S. xlsx). Finally, significant associations $(p<0.05)$ between microRNAs and the mTOR pathway from the KEGG database were chosen for further validation if $\geq 3$ microRNA target prediction tools predicted this association. Notably, as KEGG considers the mTOR pathway to be comprising both the components of the upstream Akt and PI3K pathways, we restricted the analyses only by this pathway.

Design of the microRNA-mimics. Oligonucleotides (Exiqon) for the in vivo injection were designed to be stable against nucleases, and to achieve an effective and specific incorporation of the antisense strand to the RNA-induced silencing complex (RISC) to avoid off-target effects (Elmén et al., 2005; Mook et al., 2007). The passenger sense strand was synthesized to be complementary to the antisense one to protect the molecule from endonucleases. Two sugar moieties containing a methylene bridge between the $2^{\prime}$-oxygen and the $4^{\prime}$ - carbon of the ribofuranose ring [locked nucleic acid (LNA)] were placed as $3^{\prime}$-end overhangs of both strands and additionally as the first nucleotide on the $5^{\prime}$ end of the sense strand. The latter modification serves for strand discrimination in respect to loading to the RISC. LNA-moiety restricts the flexibility of the monomer and locks it in a rigid bicyclic $\mathrm{N}$-type conformation conferring exceptional tolerance against nucleases and extremely low cellular toxicity. Moreover, these minimal modifications provide a compromise between stability and functionality both for in vitro and in vivo applications (Elmén et al., 2005; Mook et al., 2007). Scrambled control oligonucleotides (Exiqon) contain the same base modifications as the predicted microRNAs and have no homology to any known mammalian gene (www. dkfz.de/de/molekularbiologie/download/Vinnikov-et-a-S.xlsx).
Delivery of microRNA mimics to the brain. Bilateral infusions of any substances to the vicinity of ARC using metal cannulas (Plastics One) resulted in a very poor survival ( $<30 \%$, data not shown). Therefore we implemented unilateral infusion using a glass microcannula (outer/inner diameter, $100 / 58 \mu \mathrm{m}$ ), bent at $90^{\circ}$, with a beveled taper (diameter, $50 \pm$ $5 \mu \mathrm{m}$; Biomedical Instruments) to reduce tissue damage. The injection setup was assembled with a flow moderator cap as a spacer for attachment to a skull, a T-connector (inner diameter, $1 \mathrm{~mm}$; Neolab) for a stereotax holder, and an osmotic pump $(0.11 \mu \mathrm{l} / \mathrm{h}$; model \#1004, Alzet; modified from Cunningham et al., 2008) filled with the lyposomal formulation (HiPerfect reagent, Qiagen; according to the manufacturer's protocol and Zovoilis et al., 2011) of oligonucleotides in artificial CSF $(0.27 \mu \mathrm{g} / \mu \mathrm{l}$; final concentration of individual duplexes, $0.8-3.6 \mu \mathrm{M})$. For the infusion experiments, 10 of 11 predicted microRNAs were used in the mixture (omitting miR-26b). The latter had only one nucleotide difference to miR-26a and was predicted by three prediction algorithms (vs four algorithms in case of miR-26a). Implantation [coordinates relative to bregma: anteroposterior $(\mathrm{A} / \mathrm{P}),-1.46 \mathrm{~mm}$; mediolateral $(\mathrm{M} / \mathrm{L}),-0.4$; dorsoventral (D/V), $-5.5 \mathrm{~mm}$ ] was performed 3 weeks after the recombination event. Twenty-eight days later, the pumps were removed and the amount of the liquid in the pumps was measured to ensure proper pumping. Liposomal formulation of miR-103, miR-26b-FAM mimics, or scrambled oligonucleotide $(0.33 \mathrm{~mm}$ and $0.1 \mathrm{~mm}$ for highconcentration and low-concentration groups, respectively) was injected bilaterally $0.5 \mu \mathrm{l}$ per side into 4 week post-TAM DicerCKO mice using the following coordinates relative to bregma: $\mathrm{A} / \mathrm{P},-1.46 \mathrm{~mm} ; \mathrm{M} / \mathrm{L} \pm$ $0.25 \mathrm{~mm} ; \mathrm{D} / \mathrm{V},-5.75 \mathrm{~mm}$. For both ways of delivery, the mice were killed and analyzed as described above. Notably, animals revealed no signs of destruction of ARC or other hypothalamic nuclei that may potentially lead to the obesity phenotype (Xu et al., 2011).

In vitro analyses. HeLa cells were reverse-transfected by antimicroRNA siRNAs (Ambion) targeting miR-103, mir-29a, miR-26a, miR-15a, miR26b, miR-107, miR-27b, miR-93, miR-206, miR-15b, miR-320 (miProd IDs: AM10632, AM12499, AM10249, AM10235, AM12899, AM10056, AM10750, AM10951, AM10409, AM10904, AM11621, respectively). Briefly, the OptiMEM supplemented with sucrose, Lipofectamine 2000 gelatin, and fibronectin (Life Technologies) were used to coat the plates before vacuum centrifugation. The final concentration of each siRNA was $121.3 \mathrm{fmol} / \mu \mathrm{l}$. Untreated cells, supplemented with insulin-actrapid or rapamycin, were used as controls. At least three replicates per group were used. Cells were plated at a density $100,000 / \mathrm{ml}$ in $100 \%$ AIM V supplemented with pyruvate (Life Technologies). After $48 \mathrm{~h}$, they were fixed and stained for pS6RP. Image acquisition was performed at two wavelengths (DAPI/AlexaFluor488) using an Olympus Biosystems IX81 ScanR screening station and a $10 \times$ objective lens. Automated focusing was performed on DAPI-stained DNA and nine subpositions per well were acquired. For image analysis, a fully automated image processing pipeline has been developed using the KNIME (Konstanz Information Miner, Knime; RRID: nlx_151666) data mining platform with integrated image processing toolbox. After background correction of the 12-bit raw image (tif-file) using a rolling ball function, a global threshold using Otsu's method was applied to obtain binary images of the nucleus channel (DAPI). To reliably correlate segments with nuclei, a watershed function was used to divide clusters, which resulted in separated segments of objects for further processing. Edge objects were excluded. After labeling the binary image, objects were classified as nuclei if they fulfilled diverse quality control criteria based on object size, shape descriptors, and object intensity to consider only segments representing nuclei. The nuclei segments were used to generate masks for Voronoi-based segmentation of the pS6RP cytoplasm channel (AlexaFluor488). This enabled us to calculate the intensity values of the pS6RP signal for each of the nuclei.

Statistical analyses. Weight and food intake in DicerCKO and DicerCKO $^{4 \times \mathrm{TAM}}$ animals, weight in DicerCKO mice continuously infused by microRNA-mimics/scrambled oligonucleotides, weight in Dicer $^{\mathrm{fl} / \mathrm{fl}}$ females injected with $\mathrm{rAAV}$-Cre to different nuclei, weight in pair-fed experiments, and plasma hormones were analyzed by two-way ANOVA for repeated measurements ( $p$ and $F$ values are indicated in the Results), followed by one-way ANOVA for every time point and pairwise comparison between means adjusted by Bonferroni's post hoc test. 

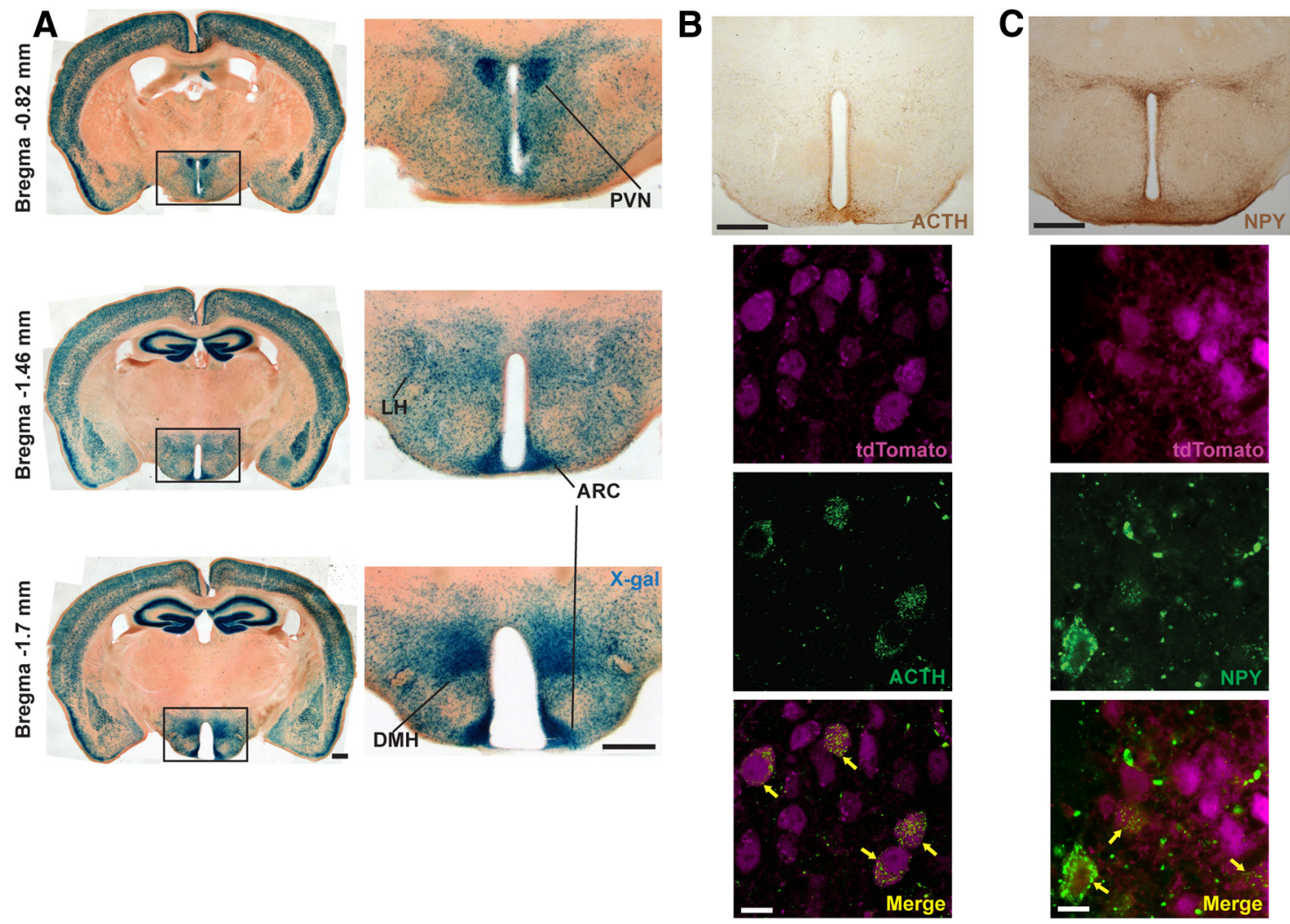

Figure 1. Recombination in the CamKCreERT2 line. $A$, X-gal staining of the forebrain in Rosa-LacZ ${ }^{\text {CaMKCreERT2 }}$ mice after 10 injections of TAM. Images on the left represent overviews of the brains at indicated levels related to the bregma. Relative hypothalamic recombination is shown on the right. $B, C$, Top, Overview of adrenocorticotropin (ACTH), a cleavage product of POMC, or NPY, immunoreactivity within the hypothalamus, respectively. Bottom, Colocalization (arrows) of ACTH or NPY signals with tdTomato in tdTomato ${ }^{\text {CaMKIIICreeRT2 }}$ line. Scale bars: $\boldsymbol{A}, \boldsymbol{B}$, top, $\boldsymbol{C}$, top, $500 \mu \mathrm{m}$; $B$, bottom, C, bottom, $10 \mu \mathrm{m}$.

Weight and food intake in the rapamycin/insulin treatment experiments were analyzed by three-way ANOVA for repeated measurements ( $p$ and $F$ values are indicated in the Results) followed by one-way ANOVA to compare all four groups for every time point and pairwise comparison between means adjusted by Bonferroni's post hoc test. The pS6RP data from DicerCKO mice were analyzed by two-way ANOVA, followed by Bonferroni's post hoc test ( $p$ and $F$ values are indicated in the Results). Food intake and pS6RP in the animals injected with oligonucleotides; and weight, food intake, and TaqMan analyses of mRNA expression in the animals injected with miR-103 and miR-26b mimic were analyzed by one-way ANOVA, followed by Newman-Keuls test ( $p$ and $F$ values are indicated in the Results). For microRNA predictions, we used the Fisher's exact test corrected by Benjamini-Hochberg procedure as described previously (Kowarsch et al., 2011). All other datasets were analyzed for statistical significance using a two-tailed unpaired Student's $t$ test ( $p$ and $t$ values are indicated in the Results). $p$ values $<0.05$ were considered significant $\left({ }^{*} p<0.05 ;{ }^{* *} p<0.01 ;{ }^{* * *} p<0.001\right)$ with respect to control groups unless otherwise stated. Data in the figures and in the text are expressed as a mean \pm SEM. Analysis was performed using GraphPad Prism (RRID: rid_000081) and Statistical Analysis System (RRID: nif0000-31484) software.

\section{Results}

Deletion of Dicer in the ARC induces hyperphagic obesity in mice

To address the role of neuronal hypothalamic noncoding RNAs for the energy homeostasis control, we deleted both alleles of the Dicer1 gene in the forebrain of adult mice (DicerCKO). In these mice, TAM-inducible recombination of the Dicer1 gene was achieved in several hypothalamic nuclei, including ARC, PVN, $\mathrm{LH}$, and DMH, as well as extra-hypothalamic tissues: BLA, cortex, and hippocampus (Fig. 1A). Using a reporter line (Madisen et al., 2010), we demonstrated that in the CaMKCreERT2 line both the cells expressing NPY/AgRP and POMC undergo recombination (Fig. $1 B, C$ ). Strikingly, both female and male homozygous DicerCKO mice gained weight rapidly compared with heterozygotes and controls (Fig. $2 A-C$; for females: genotype effect, $F_{(1,26)}=675.46, p<0.001$; interaction with time, $F_{(10,210)}=$ 43.55, $p<0.001$; for males: genotype effect, $F_{(1,17)}=238.17, p<$ 0.001 ; interaction with time, $\left.F_{(10,161)}=15.25, p<0.001\right)$. To precisely identify the region responsible for the obesity phenotype in DicerCKO animals, we inactivated the Dicer1 gene locally in various hypothalamic (ARC, DMH, LH, PVN) and extrahypothalamic (BLA; Table 1) regions via bilateral injections of the rAAV expressing Cre (rAAV-Cre) into Dicer ${ }^{\text {fl/fl }}$ mice. A weight gain (Fig. $2 D$; mean effect: $F_{(4,14)}=104.05, p<0.001$; interaction with time: $\left.F_{(64,216)}=4.69, p<0.001\right)$ was observed only if the recombined region comprised the ARC neurons, even following a precise targeting of ARC with significantly reduced amount of rAAV-Cre (Fig. $2 E ; t_{(5)}=3.38, p<0.05$ ). On the peak of the obesity phenotype ( 6 weeks after TAM treatment) in DicerCKO mice, the fat tissue mass was markedly increased, adipocytes were enlarged (Fig. 2 B, F-J; perirenal fat: $t_{(7)}=7.38, p<0.001$; perigonadal fat: $t_{(7)}=6.88, p<0.001$; fat content: $t_{(7)}=11.25, p<$ 0.001 ; body composition: $t_{(7)}=7.35, p<0.001$ ), followed by elevated plasma leptin levels (Fig. $2 K$; genotype effect: $F_{(1,8)}=$ 999.48, $p<0.001$; interaction with time: $F_{(10,74)}=57.53, p<$ $0.001)$. In the hypothalamus, levels of phosphorylated STAT3 protein, the main downstream target of leptin, were increased in the sixth week (Fig. $2 L ; t_{(6)}=3.07, p<0.05$ ). However, the sustained elevation of plasma leptin failed to reduce weight of 

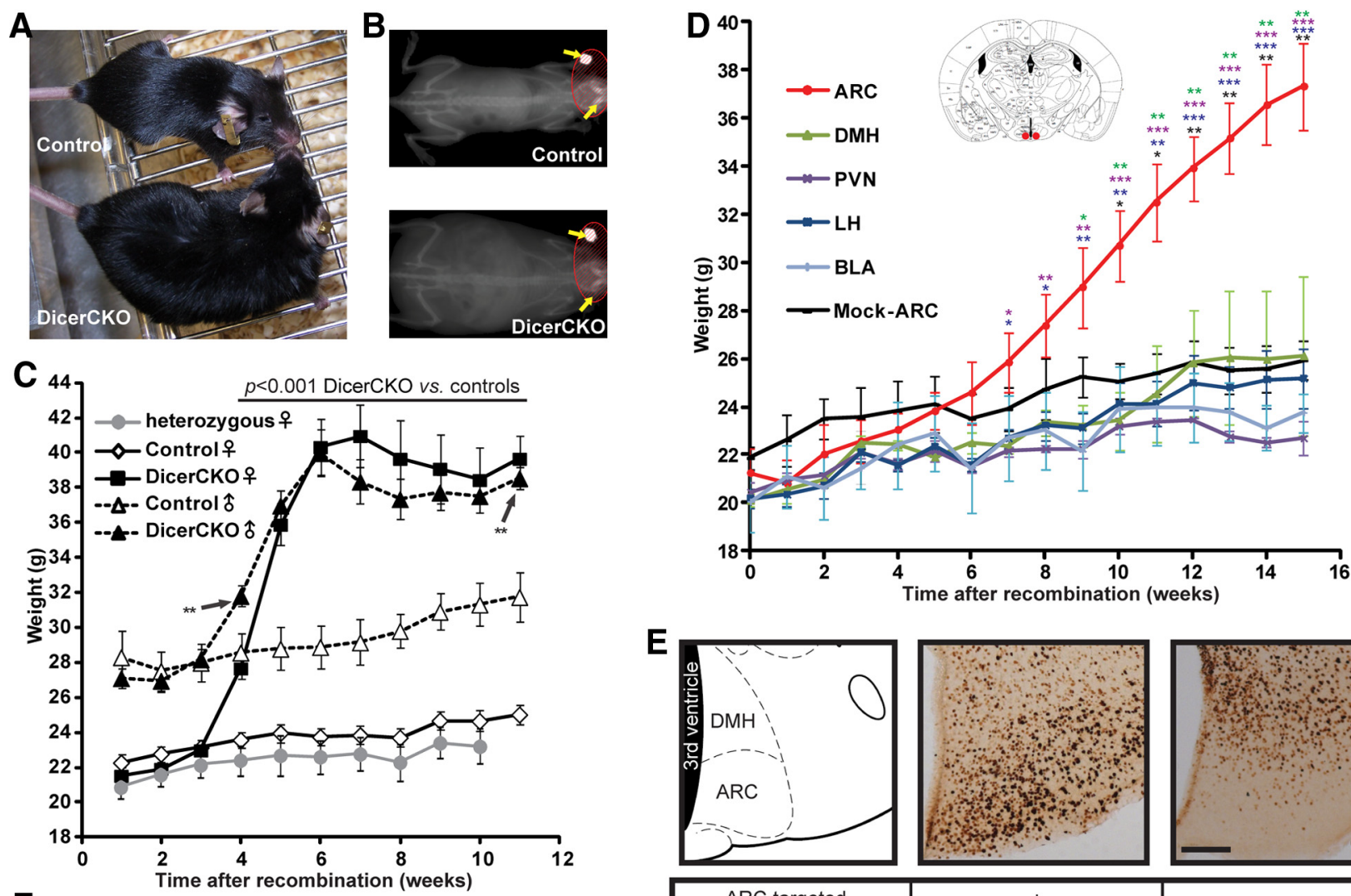

E
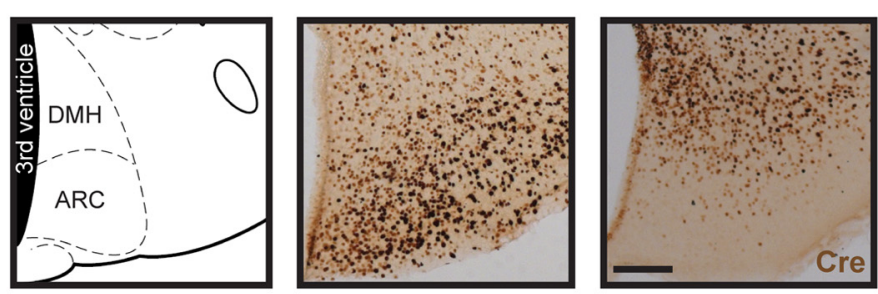

$\mathbf{F}$

Control Dicercko

\begin{tabular}{|c|c|c|}
\hline ARC targeted & + & - \\
\hline Weight gain $(g)$ & $10.2 \pm 0.8(n=5)$ & $3.7 \pm 0.1(n=2)$ \\
\hline Change to initial weight & $38.0 \pm 4.2 \%{ }^{*}$ & $14.2 \pm 0.8 \%$ \\
\hline
\end{tabular}
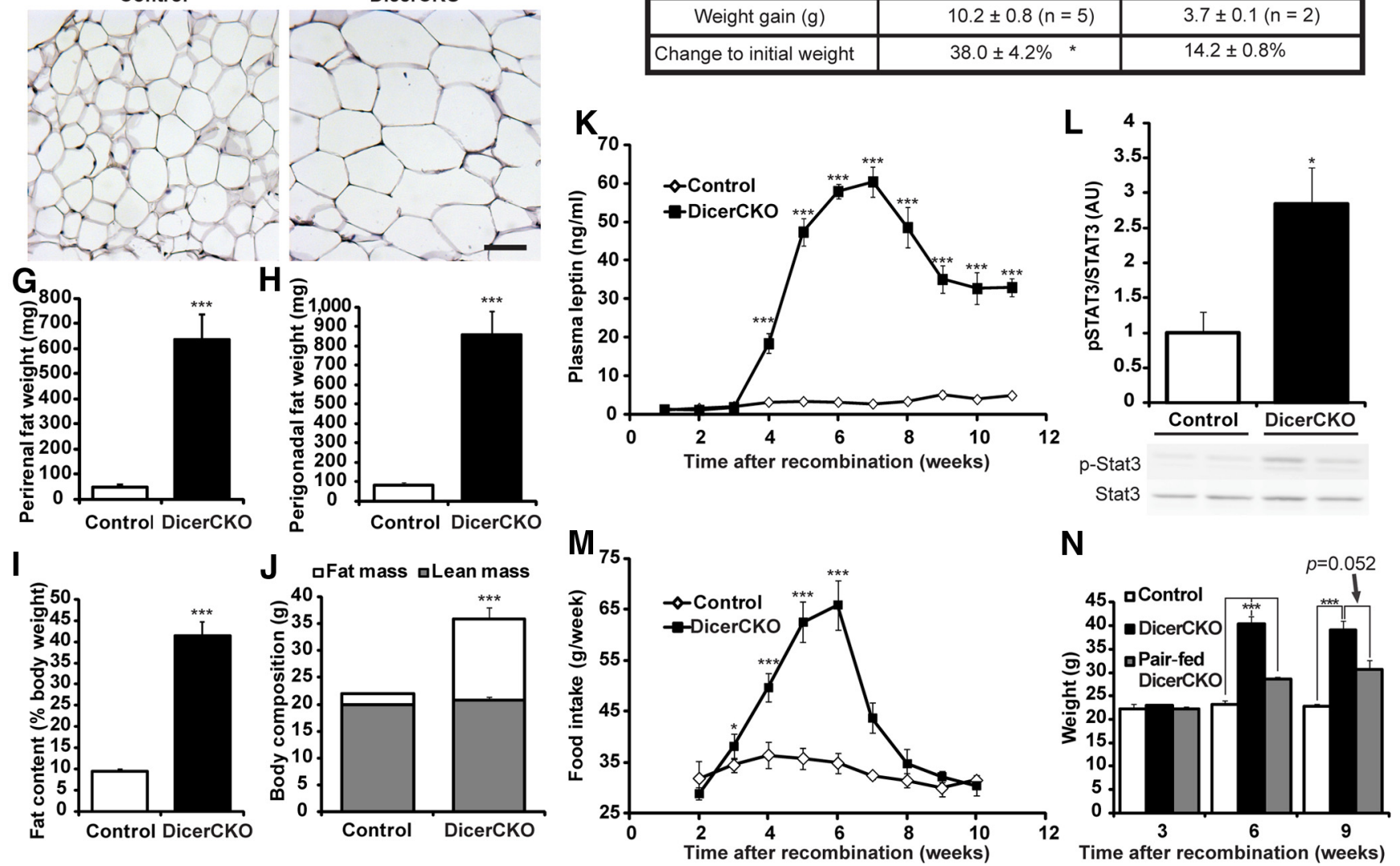

Figure 2. Dicer loss in the ARC leads to obesity. $\boldsymbol{A}, \boldsymbol{B}$, Light $(\boldsymbol{A})$ and $\mathrm{x}$-ray $(\boldsymbol{B})$ photographs of DicerCKO and control littermates 6 weeks after TAM treatment. $\boldsymbol{C}$, Weight in control, DicerCK0, and heterozygous mice. $n=14,14$, and 4, respectively, for females and $n=12$ and 7 , respectively, for males. $p$ values (separate for females and males) are outlined above the graphs unless otherwise depicted with asterisks. D, Weight of Dicer ${ }^{\text {fl/fl }}$ females, which received $140 \mathrm{nl} /$ site rAAV-Cre bilaterally into ARC (red dots in the scheme above; Paxinos and Franklin, 2001) and other brain regions. The differences between the ARC group and other hypothalamic nuclei groups are depicted with asterisks of respective colors. $\boldsymbol{E}$, Examples of correct (middle) and incorrect (right) targeting of ARC (atlas plane, left) by $10 \mathrm{nl}$ scale volumes of rAAV-Cre resulting in increased or unchanged body weight in males, respectively, shown by (Figure legend continues.) 
Table 1. Stereotaxic coordinates for site-specific recombination by bilateral rAAV injections $^{a}$

\begin{tabular}{lllllll}
\hline & \multicolumn{2}{l}{$\begin{array}{l}\text { Coordinates relative to } \\
\text { bregma }(\mathrm{mm})^{b}\end{array}$} & & \multicolumn{2}{l}{$\begin{array}{l}\text { Number of } \\
\text { animals }\end{array}$} \\
\cline { 2 - 3 } Target region & A/P & M/L & D/V & & rAAV-Cre & Mock \\
\hline ARC & & & & & \\
$\quad$ Single injection/side & -1.46 & +0.25 and -0.25 & -5.5 & & 4 & 3 \\
\multicolumn{1}{c}{ Multiple injections/side } & -2.54 & +0.25 and -0.25 & -5.5 & & 5 & \\
& -2.3 & & -5.5 & & \\
& -2.06 & & -5.5 & & \\
& -1.82 & & -5.75 & & \\
& -1.46 & & -5.75 & & \\
DMH & -1.7 & +0.4 and -0.4 & -4.9 & 3 & \\
PVN & -0.82 & +0.25 and -0.25 & -4.6 & & 4 & \\
LH & -1.46 & +1.1 and -1.1 & -4.75 & & 5 & \\
BLA & -1.34 & +3 and -3 & -4.4 & 2 & \\
\hline
\end{tabular}

${ }^{a}$ Presented are hypothalamic and extrahypothalamic regions, coordinates for stereotaxic injection, and the number of animals per group.

${ }^{b}$ All injections were done bilaterally.

DicerCKO mice, despite the normalization of food intake (Fig. $2 C, M$; for food intake: genotype effect, $F_{(1,9)}=43.08, p<0.001$; interaction with time, $\left.F_{(8,66)}=8.64, p<0.001\right)$. A contribution of the elevated food consumption in the obesity phenotype in DicerCKO mice was proven by pair-fed experiment (Fig. $2 N$; mean effect, $F_{(2,19)}=56.29, p<0.001$; interaction with time, $\left.F_{(4,27)}=13.49, p<0.001\right)$. To exclude a possibility that the observed phenotype is a consequence of cell death in the brain due to Dicer loss, we crossed DicerCKO mice with a Cre-responsive reporter line expressing tdTomato (Madisen et al., 2010). Six weeks after initiation of recombination, we observed a similar number of tdTomato-positive neurons in the ARC of DicerCKO and control animals (Fig. 3A). Moreover, TUNEL staining revealed only sporadic appearance of single apoptotic cells in ARC (Fig. 3B). Importantly, no apparent cell death was detected in the hypothalamus up to 9 months after mutation onset in contrast to a drastic loss of both: hippocampal neurons in DicerCKO animals (Fig. $3 A-G ; t_{(6)}=12.9, p<0.001$ for dentage gyrus cells) and ARC neurons after the deletion of Dicer during embryogenesis and early postnatal life (Schneeberger et al., 2012). Indeed, there was no decline in the number of POMC neurons, loss of which could cause adiposity per se (Xu et al., 2011), in the DicerCKO animals 12 weeks post-TAM, based on the quantification of the POMC-GFP-positive cells (Fig. 3C,D). This observation was supported by unchanged levels of $\alpha-\mathrm{MSH}$ at this time point ( $127.1 \pm 28.53 \%$ relative to controls, $n=4$ and 3 , respectively). Importantly, the use of an inducible CaMKII-CreERT2 bacterial artificial chromosome mouse line allowed us to study effects of microRNAs in adult mice, which was impossible previously, using constitutive Cre mouse models (Schneeberger et al., 2012). Analyses of the hypothalamic neuropeptide expression (Fig. 4;

\footnotetext{
(Figure legend continued.) Cre immunostaining. See Table 1 for additional information about injection coordinates and numbers of animals per group. $F$, Hematoxylin and eosin staining of perigonadal adipose tissue in DicerCKO and control littermates 6 weeks after recombination. $\mathbf{G}$, $\boldsymbol{H}$, Perirenal and perigonadal fat weights in DicerCKO $(n=4)$ and control $(n=5)$ animals at the same time point, respectively. I, J, Quantification of body fat content in DicerCKO $(n=4)$ and control $(n=5)$ animals measured by $x$-ray densitometry. The representative images are shown in $\boldsymbol{B}$. $\boldsymbol{K}$, Plasma leptin concentrations in DicerCKO females $(n=5)$. $\boldsymbol{L}$, Abundance of hypothalamic Tyr705-phosphorylated form of Stat3 (pStat3) protein in DicerCKO females and representative Western blot below the graph $(n=4)$. $M$, Dynamics of food intake in control and DicerCKO females ( $n=8$ and 9, respectively). $\boldsymbol{N}$, Weight of pair-fed DicerCKO females $(n=4)$. Scale bars: $\boldsymbol{E}, 250 \mu \mathrm{m} ; \boldsymbol{F}, 100 \mu \mathrm{m}$.
}

within the third week post-TAM: $t_{(10)}=2.63, p<0.05$ for $E$; fourth week: $t_{(9)}=2.58, p<0.05$ for $A ; t_{(14)}=3.45, p<0.01$ for $D ; t_{(8)}=3.36, p<0.01$ for $E$; $t_{(14)}=2.03, p=0.062$ for $F$; fifth week: $t_{(4)}=3.8, p<0.05$ for $A$; sixth week: $t_{(9)}=13.29, p<0.001$ for $A ; t_{(8)}=3.43, p<0.01$ for $B ; t_{(9)}=6.7, p<0.001$ for $C$; $t_{(8)}=$ $5.9, p<0.001$ for $D ; t_{(7)}=2.85, p<0.05$ for $E$; $t_{(8)}=2.2, p=$ 0.059 for $F$ ) revealed a discrepancy between transcripts and corresponding peptide levels, apparently resulting in an imbalance of orexigenic/anorexigenic mechanisms in DicerCKO animals. This discrepancy can be explained by the loss of microRNA-mediated suppression of translation and/or general enhancement of translation in response to the chronic overactivation of its major regulator, the mTOR kinase.

\section{The PI3K-Akt-mTOR pathway is crucial for the obesity phenotype in the DicerCKO mice}

Indeed, we found an activation of the PI3K-Akt-mTOR pathway, as measured by phosphorylation state of the $\mathrm{S} 6$ ribosomal protein (pS6RP) in ARC of DicerCKO animals (Fig. $5 A, B ; F_{(3,13)}=6.16$, $p<0.01$ ). Of note, DicerCKO animals had increased levels of insulin, the hormone known to activate the PI3K-Akt-mTOR pathway (Manning, 2004; Fig. 5C; mean effect: $F_{(2,18)}=71.91$, $p<0.001$; interaction with time: $\left.F_{(20,124)}=13.34, p<0.001\right)$. However, the DicerCKO ${ }^{4 \times \mathrm{TAM}}$ mice injected with TAM only four times within $2 \mathrm{~d}$ (compared with the typically used regimen: 10 times every $5 \mathrm{~d}$ ) did not exert any increase in insulin levels at any time points tested (Fig. 5C). On the other hand, the DicerCKO ${ }^{4 \times T A M}$ mice had a recombination pattern similar to that of DicerCKO animals, the indistinguishable hyperphagic obesity syndrome, and increased pS6 immunoreactivity in ARC (Fig. 5D-G; for weight: genotype effect, $F_{(1,11)}=44.98, p<0.001$; interaction with time, $F_{(10,110)}=3.54, p<0.001$; for food intake: genotype effect, $F_{(1,12)}=124, p<0.001$; interaction with time, $\left.F_{(9,107)}=8.48, p<0.001\right)$. To check whether insulin can aggravate hyperphagia in the DicerCKO mice, we supplemented them with an additional dose of insulin. These animals exerted only a tendency toward food-intake increase, which did not reach statistical significance if compared with untreated DicerCKO mice (Fig. $5 H$ ). We sought to explore whether overactivation of the PI3K-Akt-mTOR pathway is responsible for hyperphagia and subsequent weight gain in these mice. We treated DicerCKO animals continuously with the specific mTOR complex 1 inhibitor rapamycin, which normalized both food intake and body weight in these mice (Fig. $5 H-I$; for food intake: genotype effect, $F_{(1,18)}=$ $7.99, p<0.05$; treatment effect: $F_{(2,18)}=5.31, p<0.05$; for body weight: genotype effect, $F_{(1,16)}=32.83, p<0.001$; treatment effect, $F_{(2,16)}=30.98, p<0.001$; interaction effect between genotype and treatment: $\left.F_{(2,16)}=13.17, p<0.001\right)$. To further confirm the crucial function of the neuronal PI3K-Akt-mTOR signaling in obesity development, we inactivated a tumor suppressor and major negative regulator of this pathway, the Pten phosphatase, from the same set of neurons which were targeted in DicerCKO mice. To achieve that, we crossed Pten ${ }^{\text {fl/fl }}$ mice, previously established as an effective tool to overactivate this pathway in the adult neuronal populations (Domanskyi et al., 2011), with the CaMKCreERT2 line (PtenCKO). These mice, fed with a standard chow diet, showed a rapid development of obesity similar to DicerCKO mice, except there were sex differences in weight-gain dynamics (Fig. $5 \mathrm{~J}, \mathrm{~K}$; for females: genotype effect, $F_{(1,29)}=96.86, p<0.001$; treatment effect, $F_{(1,29)}=100.61, p<$ 0.001 ; interaction effect between genotype and treatment, $F_{(1,29)}$ $=76.76, p<0.001$; for males: genotype effect, $F_{(1,11)}=54.15, p<$ 

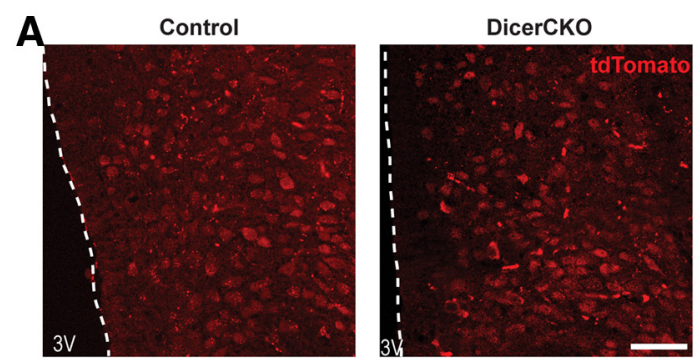

B
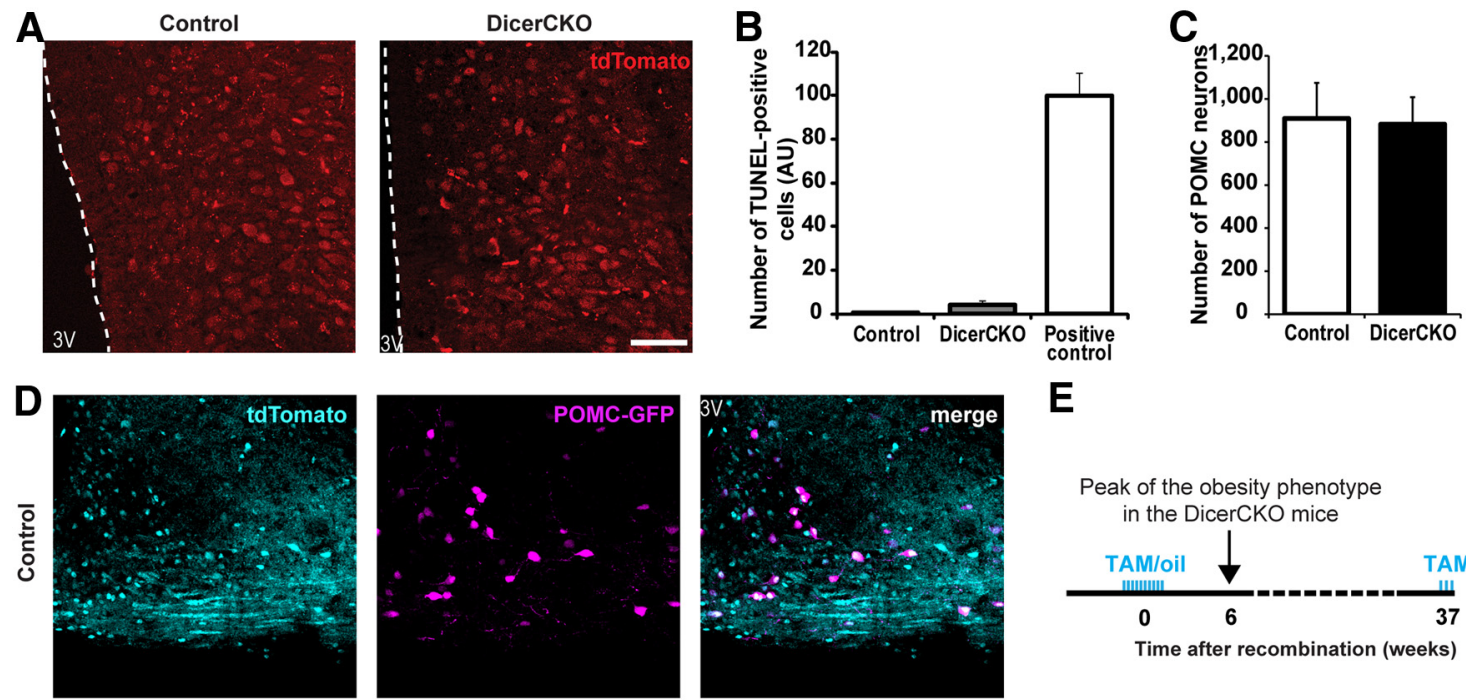

E

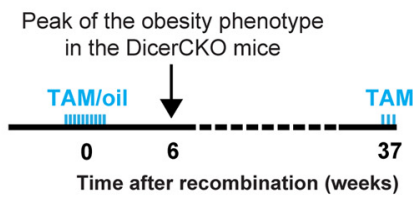

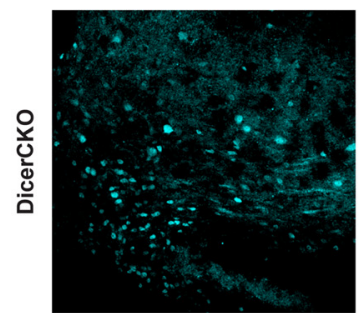

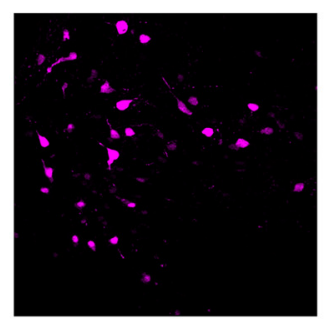

G
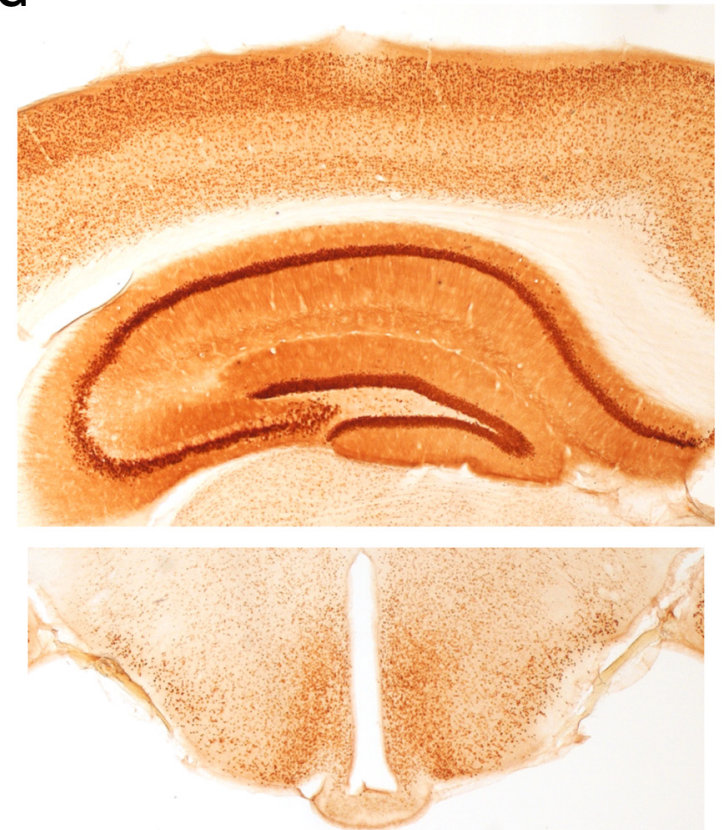

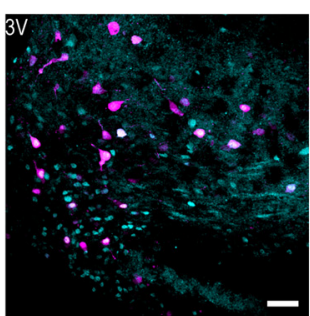

DicerCKO injected with TAM
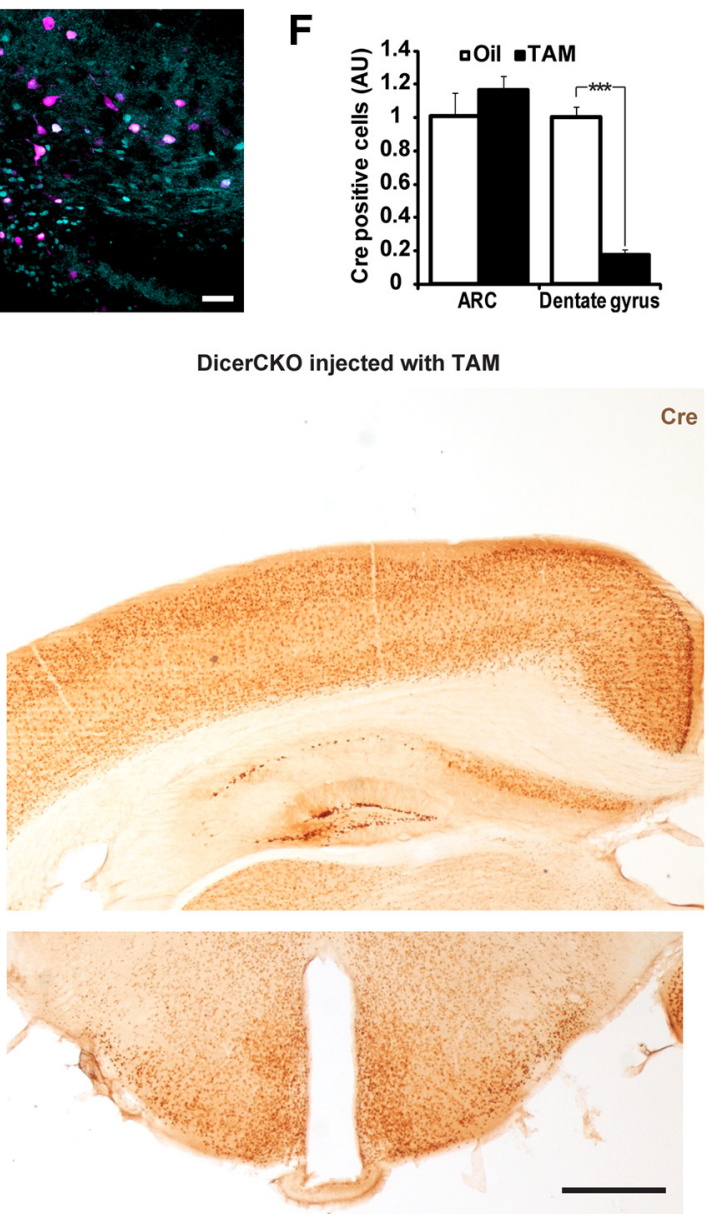

Figure 3. The ARC is preserved in DicerCK0 mice. $A$, Tomato reporter-positive cells in the ARC of tdTomato/Dicer ${ }^{\text {fl/fl }}$ (DicerCK0) or tdTomato/Dicer ${ }^{+/ f l}$ (control) animals on the background of CaMKCreERT2 6 weeks after TAM treatment in the ARC. $B$, Quantification of TUNEL-positive cells in the ARC of the DicerCKO mice at 6 weeks after TAM treatment in relation to the same area of striatal tissue of the neurodegeneration model mice used as a positive control (Kreiner et al., 2013). C, D, Quantification ( $C$ ) and representative microphotographs (D) of the P0MC-positive neurons number in ARC of POMC-GFP transgenic mice on the background of DicerCKO or control lineages 12 weeks post-TAM $(n=2)$. $E$, To visualize nuclear $C$ re, all mice received three additional injections of TAM 28,24 , and $6 \mathrm{~h}$ before being killed. $\boldsymbol{F}$, While prominent decline in (re immunosignal was observed in the hippocampus ( 9 months after initial TAM treatment), no apparent decrease in a number of Cre-positive neurons occurred in ARC and adjacent hypothalamic regions. $\mathbf{G}$, Representative staining for Cre recombinase in DicerCK0 mice initially injected by oil (control, left, $n=3$ ) or TAM (right, $n=5$ ) shown in $\boldsymbol{F}$. 3V, Third ventricle of the brain. Scale bars: $\boldsymbol{A}, 50 \mu \mathrm{m}: \boldsymbol{D}, 40 \mu \mathrm{m} ; \boldsymbol{G}, 500 \mu \mathrm{m}$.

0.001 ; interaction with time, $\left.F_{(1,11)}=46.40, p<0.001\right)$ as reported previously in a study with high-fat diet (Plum et al., 2006), and some mice failed to gain weight (see Materials and Methods). This phenotype was normalized by rapamycin treatment similar to the DicerCKO animals (Fig. 5J). All of the above data suggest that the deletion of microRNAs leads to the chronic overactivation of the PI3K-Akt-mTOR pathway in ARC neurons, resulting in the hyperphagic obesity. 
Infusion of mTOR-addressed miRNAs attenuates development of obesity To demonstrate which noncoding RNAs are specifically involved in the observed phenotype, we sought to rescue the absence of particular microRNAs in the hypothalamus of DicerCKO mice. We combined hypothalamic microRNA transcription profiling with in silico predictions by the miTALOS tool (Kowarsch et al., 2011) to find candidate microRNAs. First, we filtered out microRNAs that are not expressed or barely abundant in the hypothalamus of C57BL/6 mice (Fig. 6A;www.dkfz.de/de/molekularbiologie/ download/Vinnikov-et-al-S.xlsx). In parallel, we combined different prediction algorithms and identified candidate microRNAs putatively targeting multiple members of the PI3K-Akt-mTOR pathway. Using this strategy, we reduced the number of candidates to several that were both highly abundant in the hypothalamus and predicted to target the PI3K-AktmTOR signaling cascade by $\leq 3$ different algorithms (Fig. 6A; www.dkfz.de/de/ molekularbiologie/download/Vinnikovet-al-S.xlsx). To guarantee a constant supply of microRNA mimics and minimal damage to the tissue, we implanted beveled-tip glass microcannulas, connected to osmotic pumps, unilaterally into ARC of DicerCKO mice (Fig. 6B-D). We found that a continuous delivery of the mixture of 10 microRNA mimics starting from the third week post-TAM reduced pS6RP immunoreactivity in the hypothalamus (Fig. $7 A, B ; F_{(2,11)}=10.9$, $p<0.01)$. Accordingly, such microRNAmimics treatment attenuated both fat content and weight gain, as well as food intake, in DicerCKO mice, suggesting a functional involvement of these microRNAs and their predicted or validated (Trajkovski et al., 2011) targets in the obesity phenotype of DicerCKO animals (Fig. 7C-G; www.dkfz.de/de/molekularbiologie/download/ Vinnikov-et-al-S.xlsx; for perirenal fat, $t_{(12)}=2.27, p<0.05$; for perigonadal fat, $t_{(12)}=2.84, p<0.05$; for weight mean effect, $F_{(2,32)}=221.24, p<0.001$; interaction with time, $F_{(58,830)}=3.95$, $p<0.001$; for food intake, $\left.F_{(2,25)}=7.02, p<0.01\right)$.

Inhibition of miR-103 potently activates pS6RP signal in vitro As the mixture of microRNA candidates was validated to be efficient in vivo, we tested in vitro whether a removal of a single microRNA is sufficient to activate the PI3K-Akt-mTOR signaling. To recapitulate the insulin-independent conditions for the activation of this pathway in vitro, we performed a series of experiments on the HeLa cell line using a serum-free AIM V medium to exclude the upstream interference with the explored pathway. We reverse-transfected HeLa cells with individual antimicroRNAs. As control, we used untransfected cells treated with either insulin or rapamycin and untreated cells (Fig. $8 A, B$ ). Strikingly, similarly to the loss of Dicer in the ARC, the anti-miR-103, but none of the other 10 anti-miRs predicted by the miTALOS,

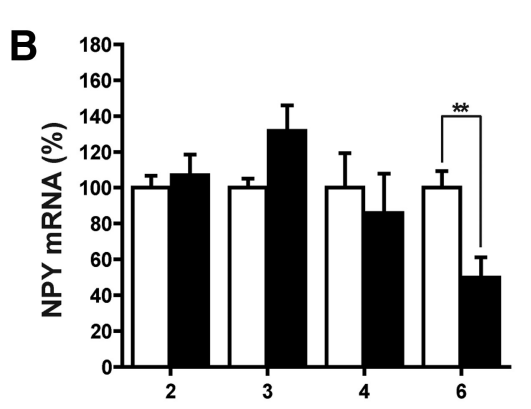

Time after recombination (weeks)

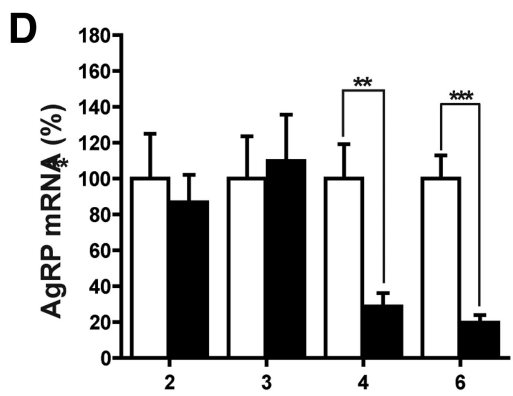

Time after recombination (weeks)

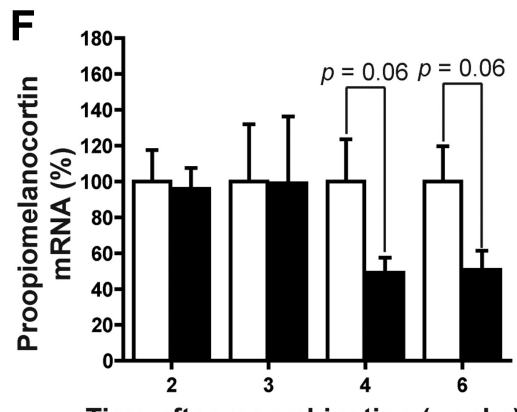

Time after recombination (weeks)

\section{DicerCKO}

\section{Control}

Figure 4. Expression of hypothalamic neuropeptides in DicerCKO mice. $\boldsymbol{A}-\boldsymbol{F}$, Levels of NPY protein $(\boldsymbol{A})$ and mRNA $(\boldsymbol{B})$; $\operatorname{AgRP}(\boldsymbol{C}$ and its mRNA $(\boldsymbol{D})$; melanocyte-stimulating hormone $\alpha$ protein, a cleavage product of adrenocorticotropin and POMC $(\boldsymbol{E})$, and POMC mRNA $(\boldsymbol{F})$ in the hypothalami of DicerCKO mice at specified time points $(n=3-9)$.

significantly upregulated pS6RP to the levels comparable to insulin-treated cells. This implies that in the group of tested microRNAs, miR-103 may be the major inhibitor of the PI3K-AktmTOR pathway.

\section{Bilateral injection of miR-103 mimic attenuates hyperphagic obesity}

To test this hypothesis, we injected bilaterally miR-103-mimic or scrambled oligonucleotides into 4-week-post-TAM DicerCKO and control littermates. As a negative control, we used a DicerCKO group injected with the miR-26b mimic (this microRNA did not effectively induce the pS6RP in vitro). Additionally, the fluorescein moiety was linked to the $3^{\prime}$ end of the passenger strand of the miR-26b mimic (www.dkfz.de/de/molekularbiologie/download/ Vinnikov-et-al-S.xlsx) to preserve its function. This enabled us to visualize the distribution of the oligonucleotides up to 2 weeks after injections (Fig. 8C-E). Similarly to the constant delivery of the mixture of microRNAs (Fig. 7 $A, B$ ) and in vitro experiments, miR-103 mimic, when injected bilaterally to ARC as late as 4 weeks post-TAM, effectively reversed pS6RP immunoreactivity in the 6-week-post-TAM DicerCKO mice (Fig. 9A). Notably, mir-103, but not miR-26b or scrambled oligonucleotides, atten- 

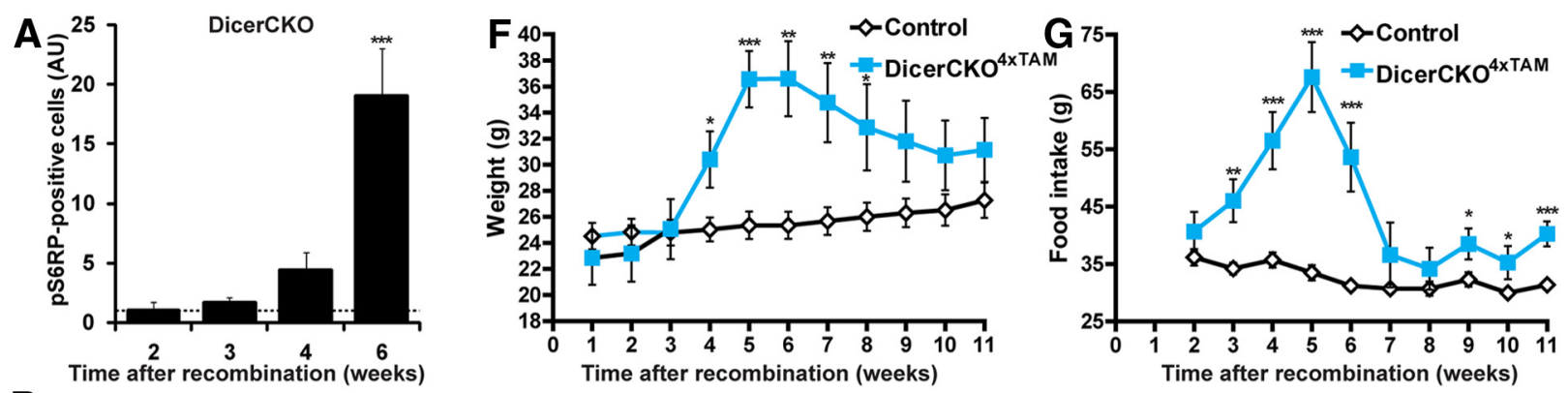

B
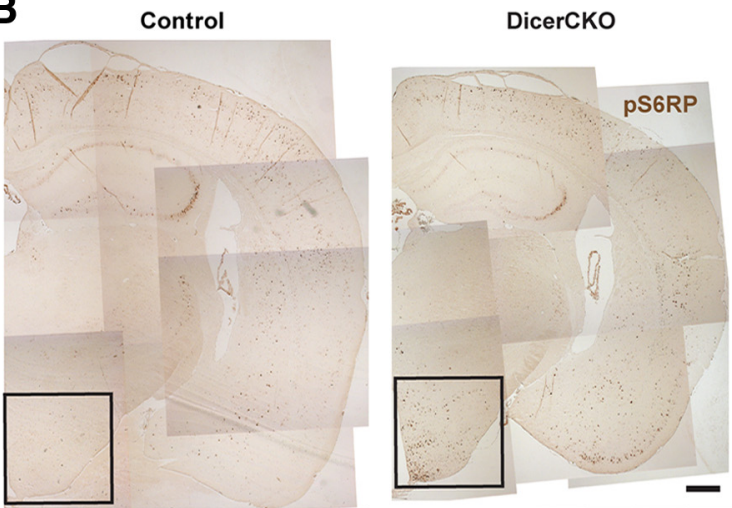

H
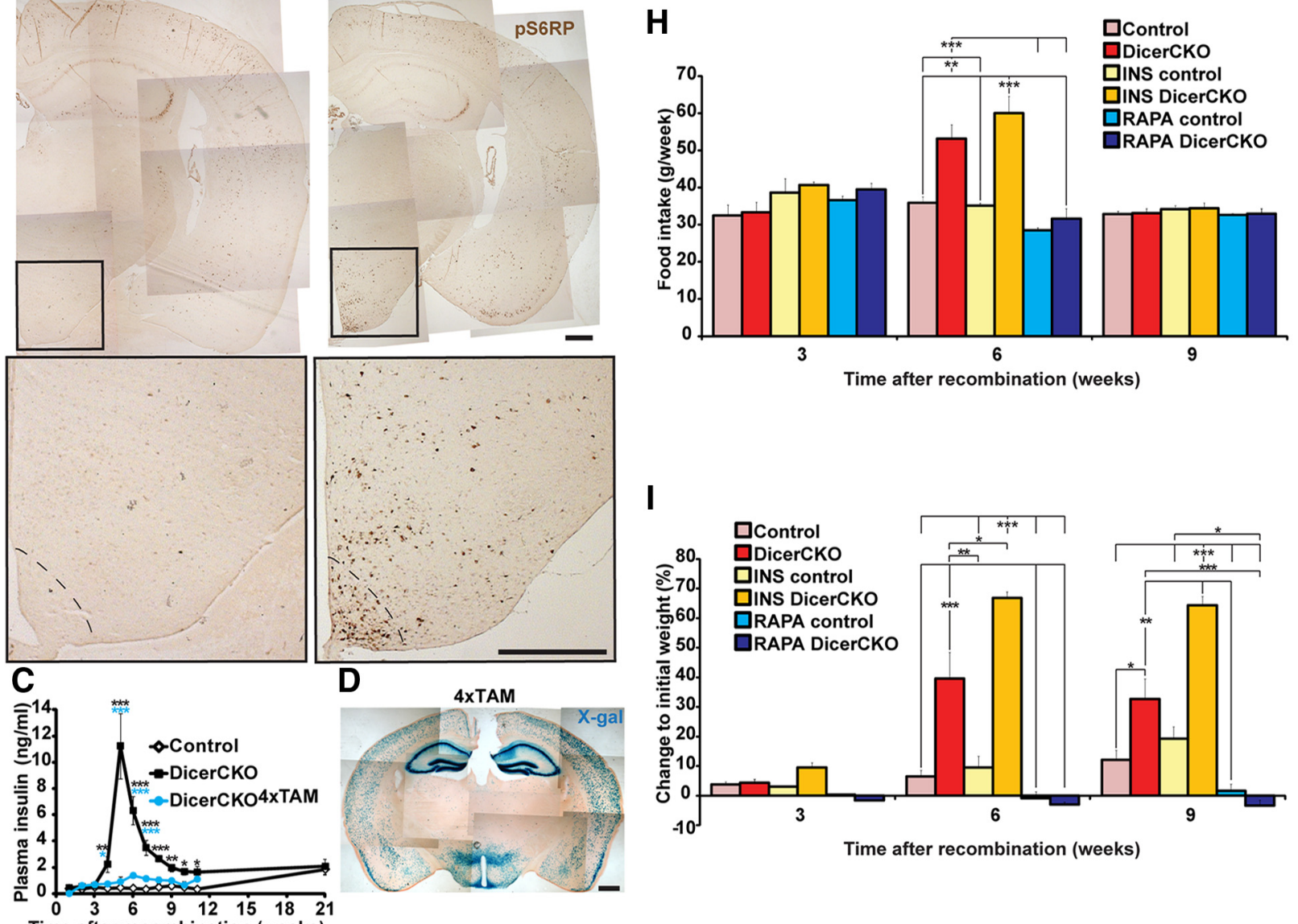

I

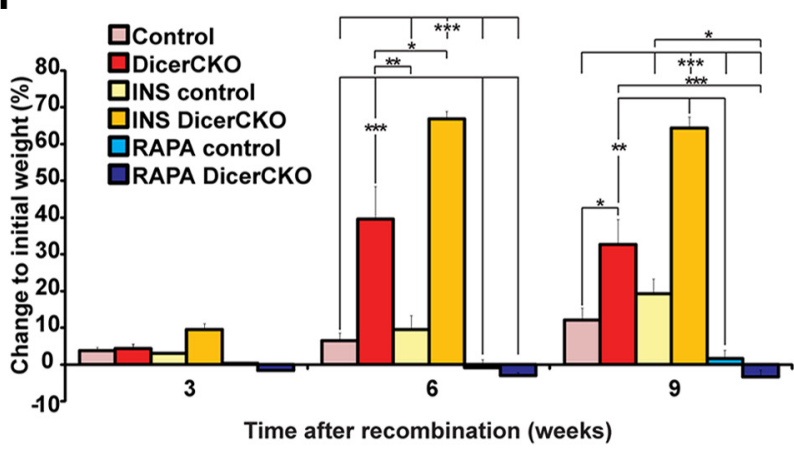

E Control

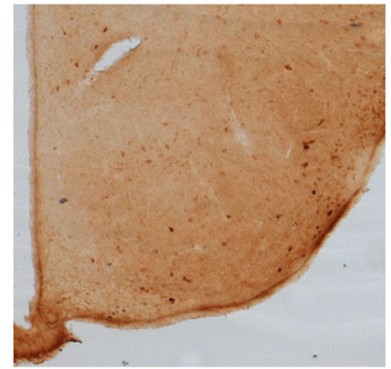

DicerCKO ${ }^{4 \times T A M}$

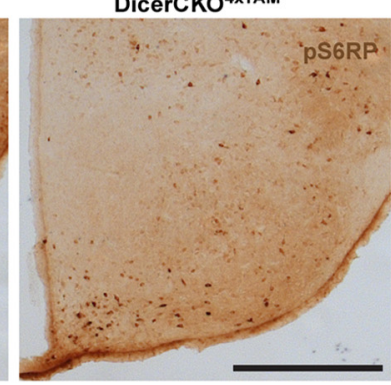

J

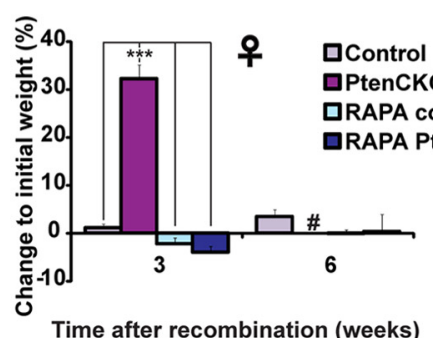

Time after recombination (weeks)
K

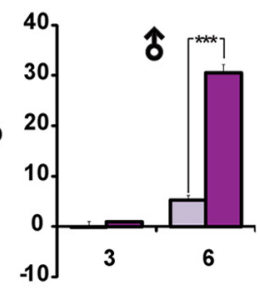

Time after recombination (weeks)

Figure 5. Enhanced PI3K-Akt-mTOR signaling causes obesity. $\boldsymbol{A}, \boldsymbol{B}$, Quantification $(\boldsymbol{A})$ and representative microphotographs $(\boldsymbol{B})$ of phospho-Ser235/236-S6 ribosomal protein (pS6RP) immunoreactivity in ARC of DicerCKO mice 6 weeks post-TAM $(n=4)$. C, Plasma insulin levels in the mice treated with 4 (DicerCKO ${ }^{4 \times \text { TAM }}$ ) or 10 (DicerCKO) injections of TAM and controls $(n=10,5$, and 5, respectively). $\boldsymbol{D}, X$-gal staining of the forebrain in Rosa-LacZ CaMKCreeRT2-4×TAM mice. E, Phospho-Ser235/236-S6 ribosomal protein (pS6RP) immunoreactivity in ARC of DicerCKO ${ }^{4 \times T_{A M}}$ mice 6 weeks post-TAM. $\boldsymbol{F}$, Weight in control and DicerCKO ${ }^{4 \times T A M}$ females $\left(n=8\right.$ and 5 , respectively). $\mathbf{G}$, Food intake in control and DicerCKO ${ }^{4 \times \operatorname{TAM}}$ females $(n=9$ and 5 , respectively). $\boldsymbol{H}, \boldsymbol{I}$, Control and DicerCKO males were treated daily with either insulin (INS; $n=3$ ) or rapamycin (RAPA; $n=4$ and 3 , respectively). Food intake $(\boldsymbol{H})$ and weight $(\boldsymbol{I})$ are presented. $n=5$ and 4 for controls and DicerCKO mice in nontreated groups. $\boldsymbol{J}, \boldsymbol{K}$, Weight of control and PtenCKO female $(\boldsymbol{J}, n=8$ and 6 , respectively) and male $(\boldsymbol{K}, n=11$ and 2, respectively) mice. Additionally, control and mutant females were treated with RAPA ( $n=9$ and 10, respectively). \#, All PtenCKO females died between third and sixth weeks after TAM treatment. Scale bars: $\boldsymbol{B}, \mathbf{D}, \boldsymbol{E}, 500 \mu \mathrm{m}$. 
uated both weight and food intake in $\mathrm{Di}$ cerCKO mice (Fig. $9 B-D$; for weights: $F_{(2,13)}=22.4, p<0.001$ and $F_{(3,18)}=$ 224.97, $p<0.001$, respectively; food intake: $\left.F_{(3,18)}=38.595, p<0.001\right)$. To address a possible molecular mechanism underlying the PI3K-Akt-mTOR-related phenotype in DicerCKO mice, we performed an mRNA expression profiling from the microdissected ARC nuclei of DicerCKO and control mice. These data revealed a number of genes within the insulin-PI3K-Akt-mTOR pathway that were significantly increased in the DicerCKO mice or had a tendency toward upregulation (Fig. 9E; for Eif2s2: $t_{(4)}=3.77$, $p<0.05$; Pik3cg: $t_{(4)}=3.99, p<0.05$; Pik3r1: $t_{(4)}=4.237, p<0.05$; Pik3r2: $t_{(4)}=$ $3.19, p<0.05$; Prkaa2: $t_{(4)}=5.399, p<0.01$; $\left.U l k 2: t_{(4)}=4.342, p<0.05\right)$. Of the six significantly upregulated transcripts, we sought to further explore the ARC levels of those $\mathrm{mR}$ NAs that were predicted by miTALOS to be targets of miR-103 (www.dkfz.de/de/ molekularbiologie/download/Vinnikovet-al-S.xlsx), namely $P i k 3 c g$ and Pik3r1. To achieve that, we implemented a more sensitive TaqMan quantitative PCR method. This assay revealed that the $P i k 3 c g$ gene, in contrast to Pik3r1, was both upregulated in DicerCKO mice and attenuated in the miR-103-mimic group (Fig. $9 F, G ; F_{(2,10)}=14.79, p<0.01$ ) suggesting a direct or indirect interaction of miR-103 with this gene, predicted by miTALOS to be a putative target of this microRNA.

\section{Discussion}

Here we demonstrate that Dicer1 removal in ARC, comprising AgRP/NPY-expressing and POMC-expressing neurons, induces PI3K-Akt-mTOR-mediated obesity. The important role of transient hyperphagia in this phenotype was proven by a significantly increased food intake in both DicerCKO and DicerCKO ${ }^{4 \times \mathrm{TAM}}$ mice as early as 3 weeks post-TAM and by the pair-fed studies.

To identify the region(s) contributing to adiposity in DicerCKO mice, we implemented rAAV gene delivery to various hypothalamic nuclei and identified ARC as the only region capable of inducing obesity. The infection with large amounts of rAAV expressing Cre recombinase under the promoter lacking specificity did not allow us to target the nuclei of interest precisely. The reason for that was a very large area of infection, which comprised not only the nuclei where the injection actually aimed, but also the regions above those. Therefore, we performed the experiment with a lower amount of virus injected, which reduced the number of recombined cells in the adjacent regions dorsal to the target nuclei (such as, for example, the ventromedial hypothalamic nucleus), which could also contribute to the observed phenotype. Despite the fact that targeting was still not exclusive for the structure of interest, obesity occurred in the Dicer ${ }^{\mathrm{fl} / \mathrm{fl}}$ animals only in the case where the area with recombined cells comprised ARC

C

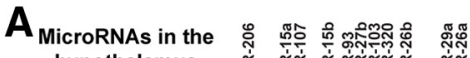

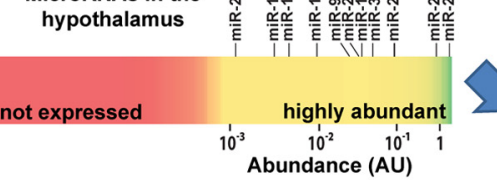

$\mathrm{mmu}-\mathrm{miR}-15 \mathrm{a} / \mathrm{b}$ mmu-miR-29a mmu-miR-103/107 mmu-miR-320 mmu-miR-26a mmu-miR-27b mmu-miR-93

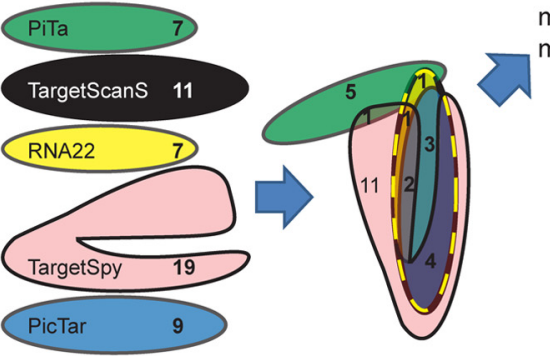
mmu-miR-206
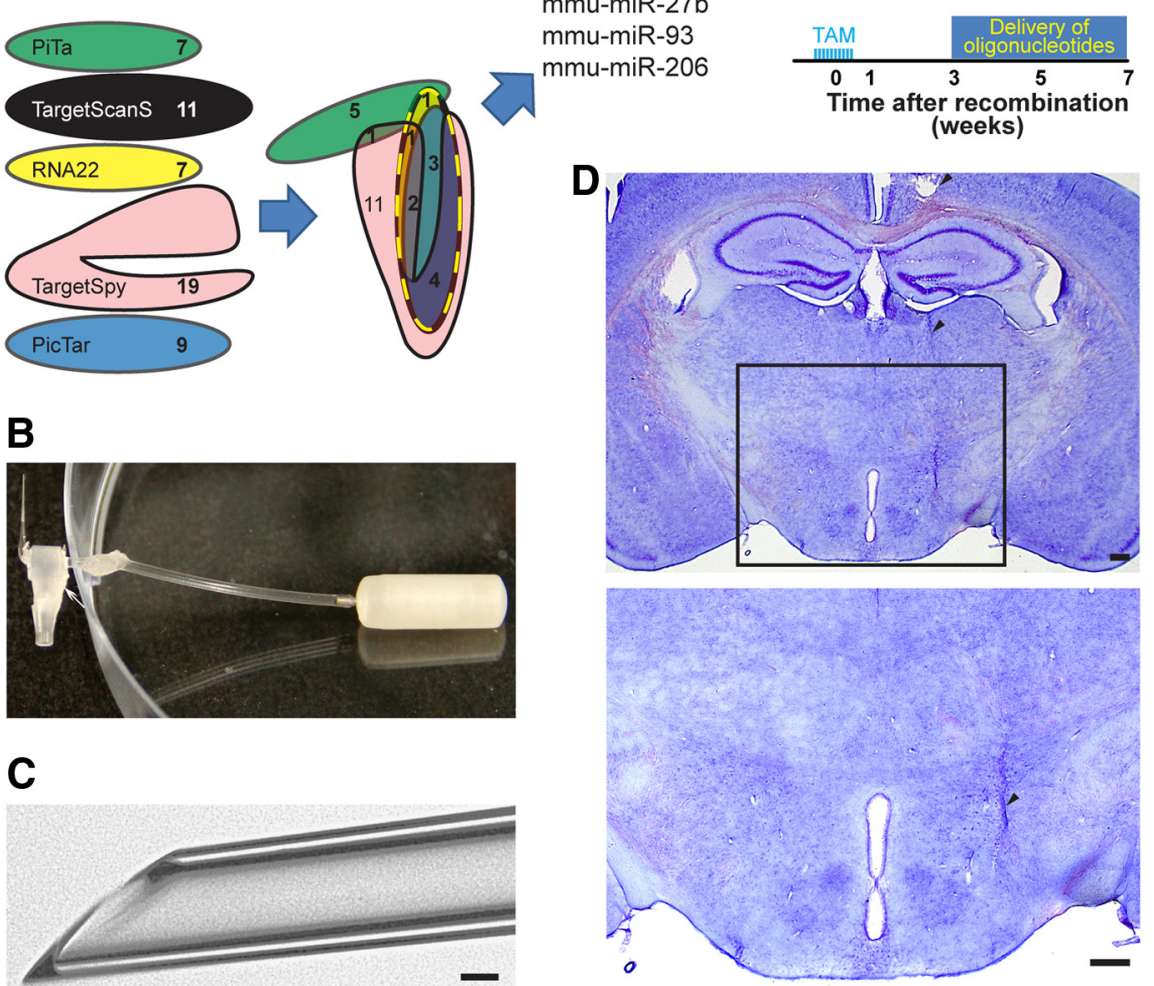
(weeks) 3'.000000000000000000000-5' 3' antisense strand \& RNA

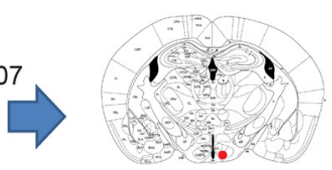

(1)

Figure 6. In silico and in vivo analyses to identify the microRNAs relevant for the DicerCKO phenotype. $A$, Workflow of candidate intification. Top left, Abundant microRNAs in the mouse hypothalamus. Bottom left, Modified Euler diagram of the $\boldsymbol{B}, \boldsymbol{C}$, Glass microcannula (taper diameter, $50 \pm 5 \mu \mathrm{m}$ ) connected to the osmotic pump $(\boldsymbol{B})$ and its beveled taper $(\boldsymbol{C})$ used to reduce damage to the tissue. The arrowhead indicates the taper of the microcannula and the arrow points to $\mathrm{a}$ T-connector attached to it. $D$, Position of a microcannula in the brain, stained by Nissl method. Arrowheads indicate microcannula track, positioned slightly lateral and dorsal to the ARC to avoid damage of this region. Scale bars: $C, 250 \mu \mathrm{m} ; \mathbf{D}, 50 \mu \mathrm{m}$.

neurons. As our targeting was not perfectly selective, we cannot rule out the cooperative effects of different nuclei (e.g., ARC with $\mathrm{DMH}$ or ventromedial hypothalamus); nor can we totally exclude the possibility that the hypothalamic nuclei, other than ARC, may induce obesity when recombined alone. Nevertheless, the rAAV allowed us to direct our in vivo microRNA mimics rescue approach to ARC, which in turn confirmed our conclusion that this nucleus contributes to the obesity phenotype.

In the hypothalamus of 4-6-week-post-TAM DicerCKO mice, we observed elevated levels of NPY, the peptide strongly stimulating hyperphagia (Erickson et al., 1996; Raposinho et al., 2001; Aponte et al., 2011; Sousa-Ferreira et al., 2011; Wu and Palmiter, 2011; Atasoy et al., 2012). Starting from the sixth week, the NPY mRNA levels in DicerCKO animals were downregulated following enhanced leptin secretion by enlarged adipocytes, thus promoting a decrease of food intake (Schwartz et al., 1996). Normalization of food intake, but not weight, in these mice suggests that the whole system could have reached the state that has been called "increased defended adiposity" (Ryan et al., 2012). These observations imply the existence of component(s) other than hy- 

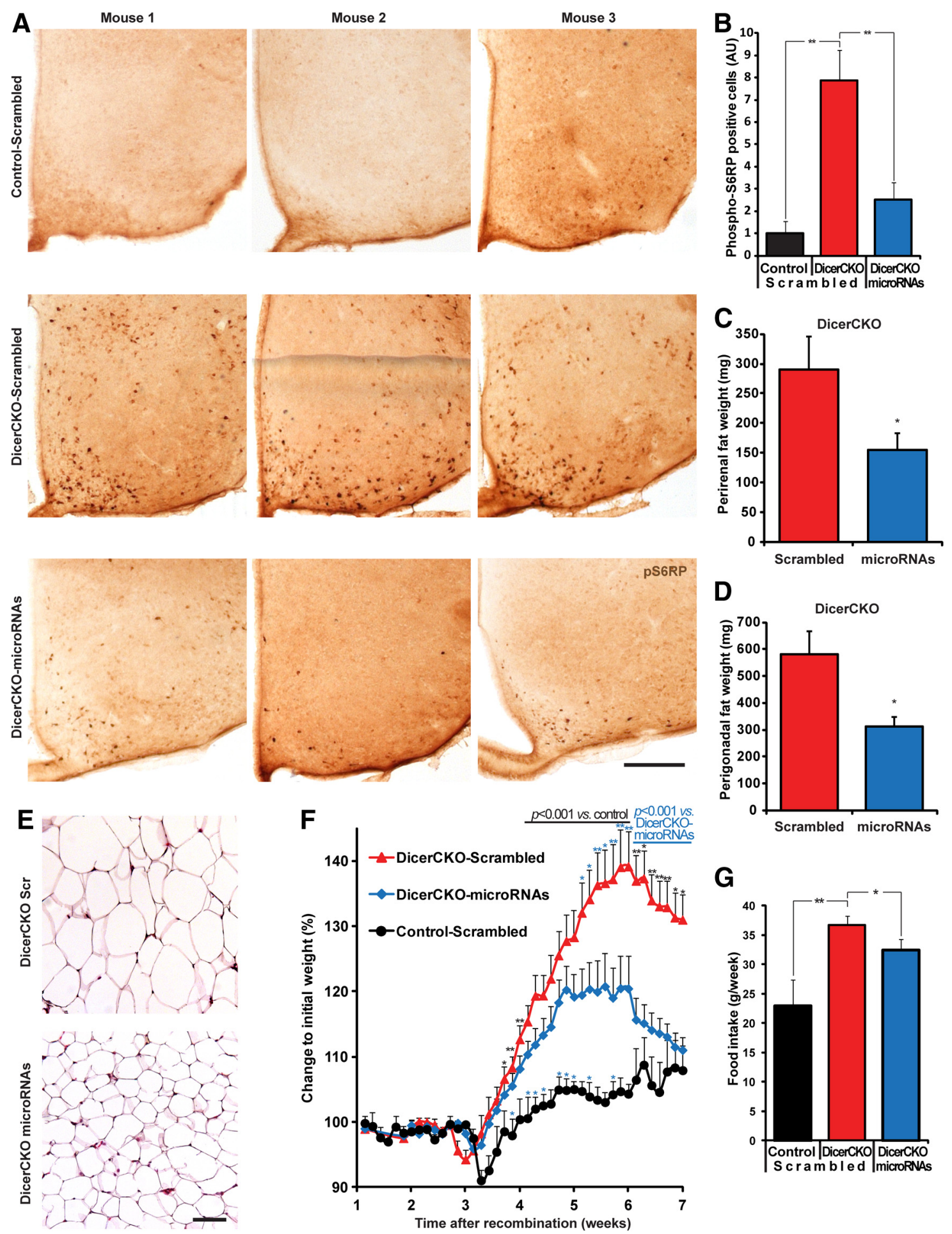

Figure 7. Specific microRNAs attenuate obesity of DicerCKO mice. A, Representative images of phospho-Ser235/236-S6 ribosomal protein (pS6RP) staining in the ARC of DicerCK0 mice 6 weeks after TAM treatment unilaterally infused by microRNA mixture or scrambled oligonucleotides. $B$, Quantification of pS6RP staining of ARC from controls and DicerCKO mice infused unilaterally with scrambled or microRNA mimics $(n=4,6$, and 4, respectively). $\boldsymbol{C}, \boldsymbol{D}$, Perirenal and perigonadal fat weights $(n=7)$. $\boldsymbol{E}$, Hematoxylin and eosin staining of perigonadal adipose tissue. $\boldsymbol{F}$, Dynamics of body weight of controls and DicerCKO mice infused unilaterally with scrambled, as well as DicerCKO mice infused with microRNA mimics to ARC ( $n=6,14$, and 15 , respectively). G, Food intake in Control-Scrambled, DicerCKO-Scrambled, and DicerCK0-microRNAs groups 4 weeks after TAM treatment ( $n=5,12$, and 11, respectively). Scale bars: $\boldsymbol{A}, 250 \mu \mathrm{m} ; \boldsymbol{E}, 100 \mu \mathrm{m}$.

perphagia stimulating obesity in DicerCKO mice, such as decreased energy expenditure or physical activity. Contrary to NPY, mRNAs of neuropeptides of melanocortin system, AgRP or $\alpha$-MSH, were decreased at 4 weeks post-TAM. Such discrepancies between mRNA and peptide levels indicate a profound im- balance between orexigenic/anorexigenic components, likely contributing to obesity of DicerCKO mice.

The kinase mTOR integrates multiple intracellular signals informing the cell about energy state. From the two mTOR complexes, a function of the mTORC1 is much better understood 

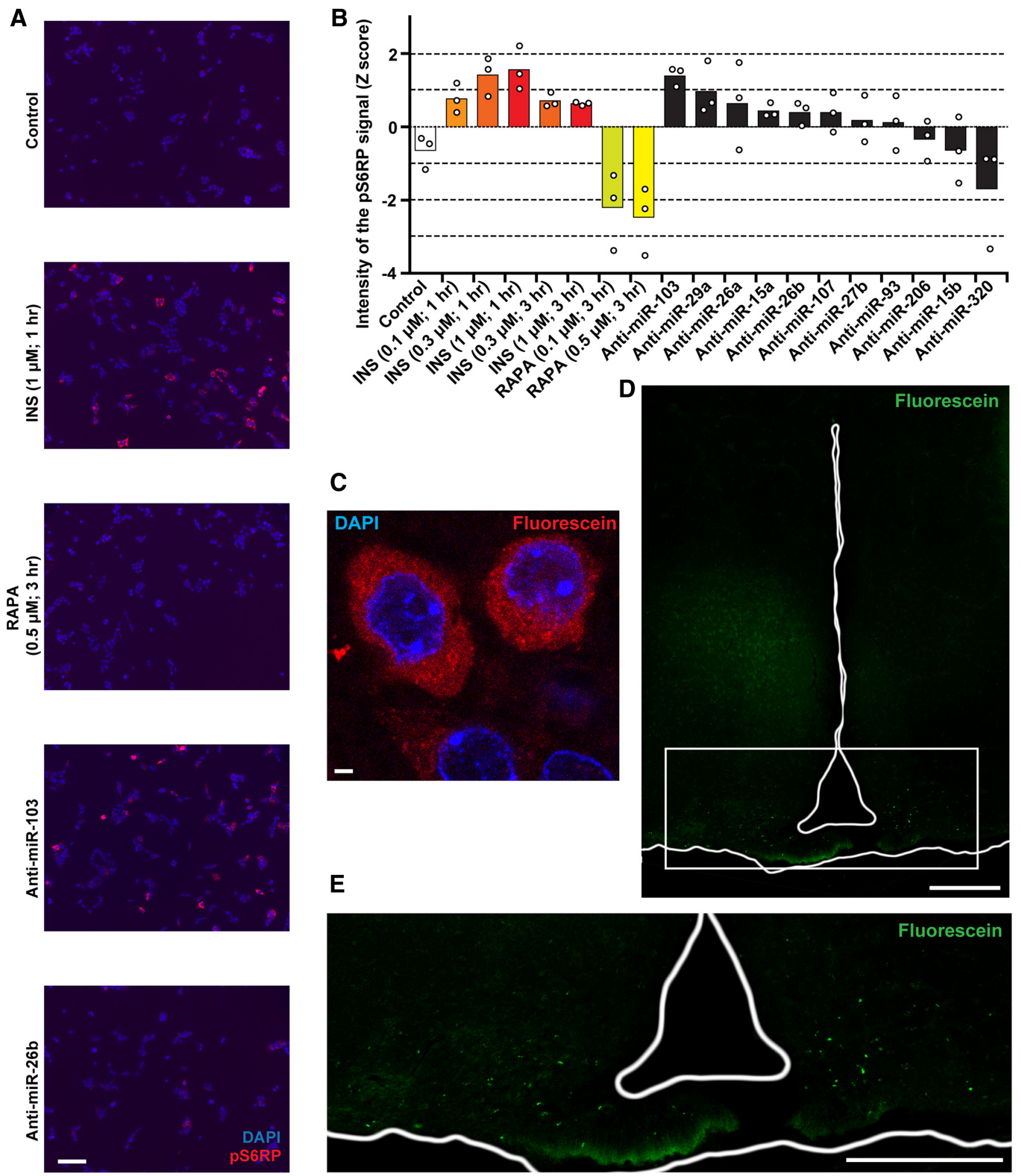

Figure 8. Modulation of microRNA expression in vitro and in vivo. $\boldsymbol{A}, \boldsymbol{B}$, Representative microphotographs $(\boldsymbol{A})$ and quantification $(\boldsymbol{B})$ of HeLa cells transfected with indicated anti-miRNAs or treated with insulin (INS) or rapamycin (RAPA; $n=3$ ). C, Incorporation of the FAM-fluorescein-labeled oligonucleotides into the neurons of the ARC $2 \mathrm{~d}$ after the injection, determined by anti-FAM antibody conjugate. $\boldsymbol{D}, \boldsymbol{E}$, Overview of the hypothalamus $(\boldsymbol{D})$ and its ventral part $(\boldsymbol{E})$ clearly showing deposition of LNA-stabilized oligonucleotides in the ARC. The signal represents direct FAM fluorescence 2 weeks after injection (i.e., 6 weeks post-TAM). In both experiments, the fluorescent moiety was attached to the $3^{\prime}$ end of the passenger strand of miR-26b. Scale bars: $\boldsymbol{A}, 100 \mu \mathrm{m} ; \boldsymbol{C}, 2 \mu \mathrm{m} ; \boldsymbol{D}$, E, $200 \mu \mathrm{m}$.

(Laplante and Sabatini, 2012). It integrates nutrient abundance and growth signals, and initiates a program for the cell to grow and proliferate, using ribosome biogenesis, protein translation, lipid biosynthesis, or inhibition of autophagy (Zoncu et al.,
2011). On the other hand, signals of the same nature modify the activity of neurons by changing the membrane potential, the amount of neurotransmitter released, and expression of neuromodulators (Wu et al., 2012). Indeed, we detected an increase in 
A
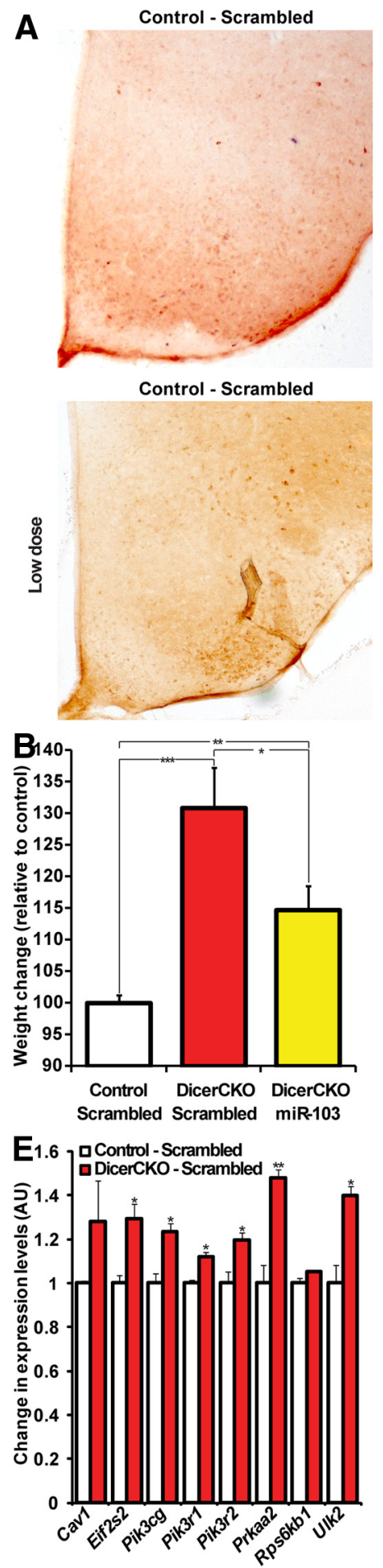
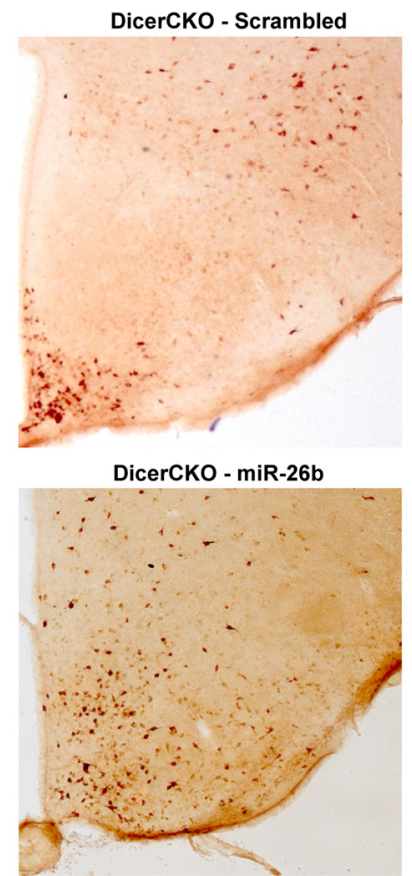

C

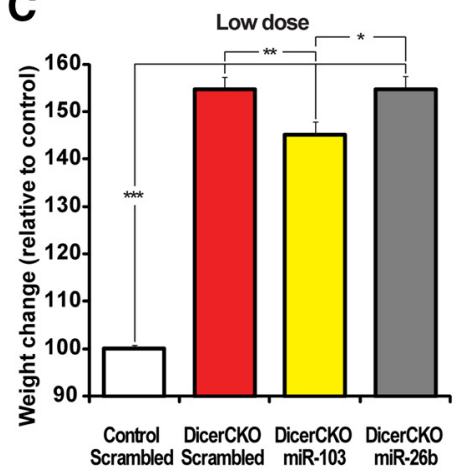

$\mathbf{F}$

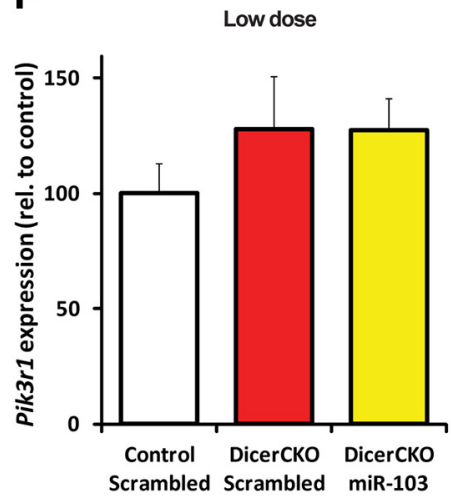

DicerCKO - miR-103

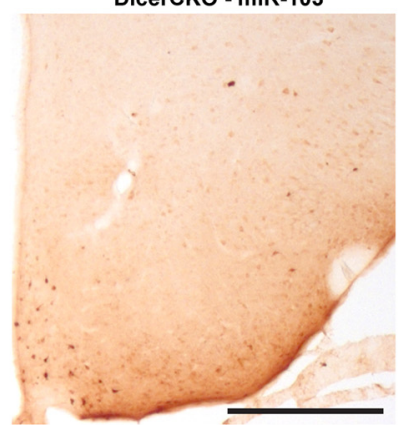

DicerCKO - miR-103

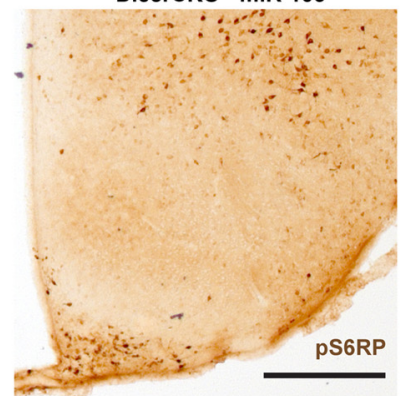

D

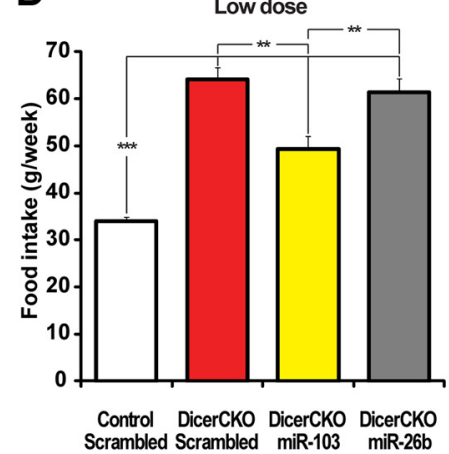

G

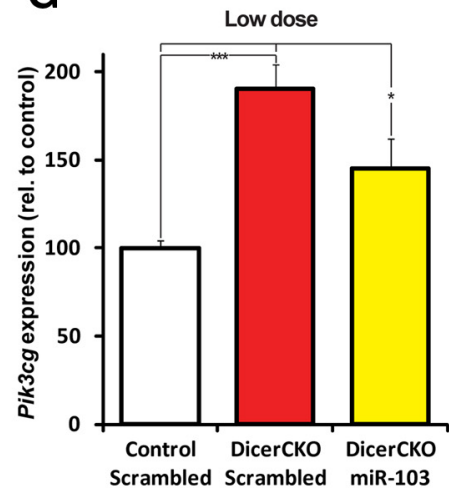

Figure 9. Delivery of miR-103 into the ARC attenuates obesity in mice. $A$, Phospho-Ser235/236-S6 ribosomal protein (pS6RP) immunoreactivity in the ARC of 6-week-post-TAM DicerCKO females bilaterally injected at 4 weeks by miR-103 or miR-26b mimics and control animals. $\boldsymbol{B}$, Weight in DicerCKO females at 5 weeks post-TAM, which were bilaterally injected to the ARC at 4 weeks post-TAM with miR-103 mimic or scrambled oligonucleotides, and control animals. $n=4,4$, and 8, respectively. $\boldsymbol{C}$, $\boldsymbol{D}$, Weight $(\boldsymbol{C})$ and food intake (D) in DicerCKO females at 6 weeks post-TAM, which were bilaterally injected to the ARC at 4 weeks with low doses of miR-103 or miR-26b mimics or scrambled oligonucleotides, and control animals. $n=5,4,4$, and 9 , respectively. E, Analysis of mRNA expression array from the microdissected ARC of DicerCKO or control mice 6 weeks post-TAM $(n=3)$. Upregulated components of the insulin-PI3K-Akt-mTOR pathway are presented. $F$, G, TaqMan analysis of the Hprt-normalized Pik3r1 (F) or Pik3cg $(\boldsymbol{G})$ expression in the microdissected ARC of 6-week-post-TAM DicerCKO mice bilaterally injected to the ARC at 4 weeks with low doses of miR-103-mimic or scrambled oligonucleotides, and control animals $(n=4,4,5)$. Scale bars: $A, 500 \mu \mathrm{m}$.

the mTOR kinase activity determined by the levels of pS6RP predominantly (but not exclusively) in ARC of DicerCKO mice. The PI3K-Akt-mTOR signaling pathway in the hypothalamic neurons is subject to activation by hormones, which transmit infor- mation about the energy state (Manning, 2004; Cota et al., 2006). A canonical view of PI3K-Akt-mTOR function in the hypothalamic neurons is that its acute enhancement reduces food intake as a reaction to nutrient availability (Cota et al., 2006). However, 
detailed analysis shows that a regulation of PI3K-Akt-mTOR activity in response to leptin and feeding may vary depending on the cell type and brain region examined (Villanueva et al., 2009). Indeed, abnormal chronic overactivation of PI3KAkt-mTOR in the hypothalamus leads to obesity (Mori et al., 2009; Yang et al., 2012).

Increase of pS6RP immunoreactivity and severe obesity syndrome accompanied by normal levels of insulin in the DicerCKO ${ }^{4 \times \text { TAM }}$ mice advocates for an intrinsic, insulin-independent driver of the PI3K-Akt-mTOR pathway overactivation. One explanation may come from a loss of certain microRNAs and a direct disinhibition of their targets within this pathway, possibly resulting in obesity. The above data suggest that insulin increase in the DicerCKO mice subjected to 10 injections of TAM may be due to Dicer loss in specific cells that were responsible for activation of insulin release (e.g., via sympathetic nervous system) and that were not recombined in DicerCKO ${ }^{4 \times \mathrm{TAM}}$ mice. To further address the role of insulin, we supplemented DicerCKO mice with additional doses of this hormone.

We observed only a tendency toward food-intake increase. At the same time, insulin, being one of the strongest anabolic signals for peripheral tissues, predictably aggravated obesity in these animals.

Previously, deletion of Pten in POMC neurons (Plum et al., 2006) has been shown to result in hyperpolarization of POMC neurons and inactivation of the anorexigenic pathway (Belgardt et al., 2009; Hill et al., 2010). Thus, we cannot exclude the possibility that POMC - neurons, as well as NPY - neurons, contribute to obesity in DicerCKO mice.

To support evidence of PI3K-Akt-mTOR-induced obesity, which was based on the increased ARC PS6RP immunoreactivity and experiments with administration of rapamycin, we performed a high-throughput mRNA profiling and TaqMan qPCR of the microdissected ARC in DicerCKO and control animals 6 weeks post-TAM. These approaches revealed within the insulinPI3K-Akt-mTOR pathway a number of genes that were upregulated in the DicerCKO mice. Keeping in mind the ubiquity of the components of the pathway and the relatively rough mode of microdissection of the ARC area (which also comprises neighboring cells that were not recombined), the presented results reflect only profound changes in transcript levels compared with the control group.

Searching for individual miRNA affecting activity of the mTOR pathway, we found that only miR-103 (but not any of the other 10 candidates) merits this requirement. In the context of metabolic control, miR-103, being highly abundant in the hypothalamus, has recently been validated to inhibit its target, Caveolin-1 (Cav1) mRNA (Trajkovski et al., 2011), a major regulator of lipid raft trafficking, which is established as a potent regulator of fat and liver homeostasis and is able to stimulate the PI3K-Akt-mTOR signaling by increasing the kinase activity of the insulin receptor and promoting phosphorylation of Akt1 (Cohen et al., 2003; Otsu et al., 2010). In our study, we also detected a tendency toward Cav1 expression increase in the microdissected ARC from DicerCKO mice 6 weeks post-TAM. As discussed above, this change in expression could well be an underestimation because of the large amount of RNA from the surrounding nonrecombined tissue present in the samples. Thus, the role of Cav1 in the central regulation of energy homeostasis remains to be addressed in the future.

A product of $P i k 3 c g$, catalytic subunit PI3K $\gamma$ (class IB PI3K or p110 $\gamma$ ), is one of the key effectors of the PI3K-Akt-mTOR cascade. In contrast to the class IA PI $3 \mathrm{~K}(\mathrm{p} 110 \alpha, \mathrm{p} 110 \beta$, and p110 $\delta$ ), this subunit interacts with either $\mathrm{p} 101$ or $\mathrm{p} 84$, but not $\mathrm{p} 85$ protein, and it has been shown to specifically mediate $G_{i}$ proteincoupled receptor (GPCRP-dependent effects (Stoyanov et al., 1995; Hawkins and Stephens, 2007). Compared with the other class I PI3Ks, its expression is not ubiquitous and is restricted to the cardiovascular and immune systems, as well as the brain, where PI3K $\gamma$ is strongly expressed in the neurons and is involved in synaptic plasticity (Kim et al., 2011; Sim et al., 2013).

Our data suggest that upregulation of the $P i k 3 c g$ transcript in response to the loss of miR-103, and possibly other microRNAs in ARC, may contribute to downstream PI3K-Akt-mTOR pathway overactivation and obesity. A detailed delineation of microRNA-target interactions, such as miR-103 and Pik3cg in a specific cellular context within the hypothalamus, is of immense importance for the understanding of (patho-)physiological mechanisms of energy homeostasis regulation by the CNS. In conclusion, the PI3K-Akt-mTOR cascade activity in the brain is known to increase with age and cause adverse effects on energy metabolism control (Yang et al., 2012; Johnson et al., 2013). On the other hand, an age-dependent decline in Dicer expression in the tissues controlling energy homeostasis (i.e., in fat tissue and globally in the brain; Mori et al., 2012) might contribute to the metabolic syndrome (Blandino et al., 2012). Therefore, our study provides a missing link between noncoding RNAs and the PI3K- 
Akt-mTOR pathway in hypothalamic neurons responsible for energy metabolism control and delineates the role of this link in obesity development (Fig. 10).

\section{Notes}

Supplemental material for this article is available at http://www.dkfz.de/ de/molekularbiologie/download/Vinnikov-et-al-S.xlsx: supplemental data, microRNA analyses. The spreadsheets contain the data of hypothalamic microRNA TaqMan qPCR array (\#1) and predictions by each of the five different algorithms that are embedded into the miTALOS tool (\#2-6). Spreadsheet \#7 outlines the strategy of shortening the candidate list. The oligonucleotides used in the study are outlined in the spreadsheet \#8. This material has not been peer reviewed.

\section{References}

Aponte Y, Atasoy D, Sternson SM (2011) AGRP neurons are sufficient to orchestrate feeding behavior rapidly and without training. Nat Neurosci 14:351-355. CrossRef Medline

Atasoy D, Betley JN, Su HH, Sternson SM (2012) Deconstruction of a neural circuit for hunger. Nature 488:172-177. CrossRef Medline

Bartel DP (2009) MicroRNAs: target recognition and regulatory functions. Cell 136:215-233. CrossRef Medline

Belgardt BF, Brüning JC (2010) CNS leptin and insulin action in the control of energy homeostasis. Ann N Y Acad Sci 1212:97-113. CrossRef Medline

Belgardt BF, Okamura T, Brüning JC (2009) Hormone and glucose signalling in POMC and AgRP neurons. J Physiol 587:5305-5314. CrossRef Medline

Blandino G, Valerio M, Cioce M, Mori F, Casadei L, Pulito C, Sacconi A, Biagioni F, Cortese G, Galanti S, Manetti C, Citro G, Muti P, Strano S (2012) Metformin elicits anticancer effects through the sequential modulation of DICER and c-MYC. Nat Commun 3:865. CrossRef Medline

Cobb BS, Nesterova TB, Thompson E, Hertweck A, O'Connor E, Godwin J, Wilson CB, Brockdorff N, Fisher AG, Smale ST, Merkenschlager M (2005) $\mathrm{T}$ cell lineage choice and differentiation in the absence of the RNase III enzyme Dicer. J Exp Med 201:1367-1373. CrossRef Medline

Cohen AW, Razani B, Wang XB, Combs TP, Williams TM, Scherer PE, Lisanti MP (2003) Caveolin-1-deficient mice show insulin resistance and defective insulin receptor protein expression in adipose tissue. Am J Physiol Cell Physiol 285:C222-C235. CrossRef Medline

Cota D, Proulx K, Smith KA, Kozma SC, Thomas G, Woods SC, Seeley RJ (2006) Hypothalamic mTOR signaling regulates food intake. Science 312:927-930. CrossRef Medline

Cunningham MG, O'Connor RP, Wong SE (2008) Construction and implantation of a microinfusion system for sustained delivery of neuroactive agents. J Vis Exp pii:716. CrossRef Medline

Domanskyi A, Geissler C, Vinnikov IA, Alter H, Schober A, Vogt MA, Gass P, Parlato R, Schütz G (2011) Pten ablation in adult dopaminergic neurons is neuroprotective in Parkinson's disease models. FASEB J 25:2898-2910. CrossRef Medline

Elmén J, Thonberg H, Ljungberg K, Frieden M, Westergaard M, Xu Y, Wahren B, Liang Z, Ørum H, Koch T, Wahlestedt C (2005) Locked nucleic acid (LNA) mediated improvements in siRNA stability and functionality. Nucleic Acids Res 33:439-447. CrossRef Medline

Elmquist JK, Elias CF, Saper CB (1999) From lesions to leptin: hypothalamic control of food intake and body weight. Neuron 22:221-232. CrossRef Medline

Erdmann G, Schütz G, Berger S (2007) Inducible gene inactivation in neurons of the adult mouse forebrain. BMC Neurosci 8:63. CrossRef Medline

Erickson JC, Hollopeter G, Palmiter RD (1996) Attenuation of the obesity syndrome of ob/ob mice by the loss of neuropeptide Y. Science 274:17041707. CrossRef Medline

Finucane MM, Stevens GA, Cowan MJ, Danaei G, Lin JK, Paciorek CJ, Singh GM, Gutierrez HR, Lu Y, Bahalim AN, Farzadfar F, Riley LM, Ezzati M, Ezzati M (2011) National, regional, and global trends in body-mass index since 1980: systematic analysis of health examination surveys and epidemiological studies with 960 country-years and 9.1 million participants. Lancet 377:557-567. CrossRef Medline

Friedman JM, Halaas JL (1998) Leptin and the regulation of body weight in mammals. Nature 395:763-770. CrossRef Medline

Hawkins PT, Stephens LR (2007) PI3Kgamma is a key regulator of inflam- matory responses and cardiovascular homeostasis. Science 318:64-66. CrossRef Medline

Hill JW, Elias CF, Fukuda M, Williams KW, Berglund ED, Holland WL, Cho YR, Chuang JC, Xu Y, Choi M, Lauzon D, Lee CE, Coppari R, Richardson JA, Zigman JM, Chua S, Scherer PE, Lowell BB, Brüning JC, Elmquist JK (2010) Direct insulin and leptin action on pro-opiomelanocortin neurons is required for normal glucose homeostasis and fertility. Cell Metab 11:286-297. CrossRef Medline

Isermann B, Vinnikov IA, Madhusudhan T, Herzog S, Kashif M, Blautzik J, Corat MA, Zeier M, Blessing E, Oh J, Gerlitz B, Berg DT, Grinnell BW, Chavakis T, Esmon CT, Weiler H, Bierhaus A, Nawroth PP (2007) Activated protein $\mathrm{C}$ protects against diabetic nephropathy by inhibiting endothelial and podocyte apoptosis. Nat Med 13:1349-1358. CrossRef Medline

Johnson SC, Rabinovitch PS, Kaeberlein M (2013) mTOR is a key modulator of ageing and age-related disease. Nature 493:338-345. CrossRef Medline

Jordan SD, Krüger M, Willmes DM, Redemann N, Wunderlich FT, Brönneke HS, Merkwirth C, Kashkar H, Olkkonen VM, Böttger T, Braun T, Seibler J, Brüning JC (2011) Obesity-induced overexpression of miRNA-143 inhibits insulin-stimulated AKT activation and impairs glucose metabolism. Nat Cell Biol 13:434-446. CrossRef Medline

Kanehisa M, Araki M, Goto S, Hattori M, Hirakawa M, Itoh M, Katayama T, Kawashima S, Okuda S, Tokimatsu T, Yamanishi Y (2008) KEGG for linking genomes to life and the environment. Nucleic Acids Res 36:D480 D484. Medline

Kellendonk C, Tronche F, Casanova E, Anlag K, Opherk C, Schütz G (1999) Inducible site-specific recombination in the brain. J Mol Biol 285:175182. CrossRef Medline

Kim JI, Lee HR, Sim SE, Baek J, Yu NK, Choi JH, Ko HG, Lee YS, Park SW, Kwak C, Ahn SJ, Choi SY, Kim H, Kim KH, Backx PH, Bradley CA, Kim E, Jang DJ, Lee K, Kim SJ, et al. (2011) PI3Kgamma is required for NMDA receptor-dependent long-term depression and behavioral flexibility. Nat Neurosci 14:1447-1454. CrossRef Medline

Knobloch HS, Charlet A, Hoffmann LC, Eliava M, Khrulev S, Cetin AH, Osten P, Schwarz MK, Seeburg PH, Stoop R, Grinevich V (2012) Evoked axonal oxytocin release in the central amygdala attenuates fear response. Neuron 73:553-566. CrossRef Medline

Konopka W, Kiryk A, Novak M, Herwerth M, Parkitna JR, Wawrzyniak M, Kowarsch A, Michaluk P, Dzwonek J, Arnsperger T, Wilczynski G, Merkenschlager M, Theis FJ, Köhr G, Kaczmarek L, Schütz G (2010) MicroRNA loss enhances learning and memory in mice. J Neurosci 30: 14835-14842. CrossRef Medline

Kornfeld JW, Baitzel C, Könner AC, Nicholls HT, Vogt MC, Herrmanns K, Scheja L, Haumaitre C, Wolf AM, Knippschild U, Seibler J, Cereghini S, Heeren J, Stoffel M, Brüning JC (2013) Obesity-induced overexpression of miR-802 impairs glucose metabolism through silencing of Hnflb. Nature 494:111-115. CrossRef Medline

Kowarsch A, Preusse M, Marr C, Theis FJ (2011) miTALOS: analyzing the tissue-specific regulation of signaling pathways by human and mouse microRNAs. Rna 17:809-819. CrossRef Medline

Kreiner G, Bierhoff H, Armentano M, Rodriguez-Parkitna J, Sowodniok K, Naranjo JR, Bonfanti L, Liss B, Schütz G, Grummt I, Parlato R (2013) A neuroprotective phase precedes striatal degeneration upon nucleolar stress. Cell Death Differ 20:1455-1464. CrossRef Medline

Laplante M, Sabatini DM (2012) mTOR signaling in growth control and disease. Cell 149:274-293. CrossRef Medline

Lesche R, Groszer M, Gao J, Wang Y, Messing A, Sun H, Liu X, Wu H (2002) $\mathrm{Cre} /$ loxP-mediated inactivation of the murine Pten tumor suppressor gene. Genesis 32:148-149. CrossRef Medline

Madisen L, Zwingman TA, Sunkin SM, Oh SW, Zariwala HA, Gu H, Ng LL, Palmiter RD, Hawrylycz MJ, Jones AR, Lein ES, Zeng H (2010) A robust and high-throughput Cre reporting and characterization system for the whole mouse brain. Nat Neurosci 13:133-140. CrossRef Medline

Manning BD (2004) Balancing Akt with S6K: implications for both metabolic diseases and tumorigenesis. J Cell Biol 167:399-403. CrossRef Medline

Mikl M, Vendra G, Doyle M, Kiebler MA (2010) RNA localization in neurite morphogenesis and synaptic regulation: current evidence and novel approaches. J Comp Physiol A Neuroethol Sens Neural Behav Physiol 196: 321-334. CrossRef Medline

Mook OR, Baas F, de Wissel MB, Fluiter K (2007) Evaluation of locked 
nucleic acid-modified small interfering RNA in vitro and in vivo. Mol Cancer Ther 6:833-843. CrossRef Medline

Mori H, Inoki K, Münzberg H, Opland D, Faouzi M, Villanueva EC, Ikenoue T, Kwiatkowski D, MacDougald OA, Myers MG Jr, Guan KL (2009) Critical role for hypothalamic mTOR activity in energy balance. Cell Metab 9:362-374. CrossRef Medline

Mori MA, Raghavan P, Thomou T, Boucher J, Robida-Stubbs S, Macotela Y, Russell SJ, Kirkland JL, Blackwell TK, Kahn CR (2012) Role of microRNA processing in adipose tissue in stress defense and longevity. Cell Metab 16:336-347. CrossRef Medline

Myers MG Jr, Münzberg H, Leinninger GM, Leshan RL (2009) The geometry of leptin action in the brain: more complicated than a simple ARC. Cell Metab 9:117-123. CrossRef Medline

Otsu K, Toya Y, Oshikawa J, Kurotani R, Yazawa T, Sato M, Yokoyama U, Umemura S, Minamisawa S, Okumura S, Ishikawa Y (2010) Caveolin gene transfer improves glucose metabolism in diabetic mice. Am J Physiol Cell Physiol 298:C450-C456. CrossRef Medline

Paxinos G, Franklin KBJ (2001) The mouse brain in stereotaxic coordinates, 2nd edition. San Diego: Academic.

Plum L, Ma X, Hampel B, Balthasar N, Coppari R, Münzberg H, Shanabrough M, Burdakov D, Rother E, Janoschek R, Alber J, Belgardt BF, Koch L, Seibler J, Schwenk F, Fekete C, Suzuki A, Mak TW, Krone W, Horvath TL, et al. (2006) Enhanced PIP3 signaling in POMC neurons causes KATP channel activation and leads to diet-sensitive obesity. J Clin Invest 116:1886-1901. CrossRef Medline

Raposinho PD, Pierroz DD, Broqua P, White RB, Pedrazzini T, Aubert ML (2001) Chronic administration of neuropeptide Y into the lateral ventricle of C57BL/6J male mice produces an obesity syndrome including hyperphagia, hyperleptinemia, insulin resistance, and hypogonadism. Mol Cell Endocrinol 185:195-204. CrossRef Medline

Ryan KK, Woods SC, Seeley RJ (2012) Central nervous system mechanisms linking the consumption of palatable high-fat diets to the defense of greater adiposity. Cell Metab 15:137-149. CrossRef Medline

Schneeberger M, Altirriba J, García A, Esteban Y, Castaño C, GarcíaLavandeira M, Alvarez CV, Gomis R, Claret M (2012) Deletion of miRNA processing enzyme Dicer in POMC-expressing cells leads to pituitary dysfunction, neurodegeneration and development of obesity. Mol Metab 2:74-85. CrossRef Medline

Schratt G (2009) MicroRNAs at the synapse. Nat Rev Neurosci 10:842-849. CrossRef Medline

Schwartz MW, Seeley RJ, Campfield LA, Burn P, Baskin DG (1996) Identification of targets of leptin action in rat hypothalamus. J Clin Invest 98:1101-1106. CrossRef Medline

Seeley RJ, Woods SC (2003) Monitoring of stored and available fuel by the CNS: implications for obesity. Nat Rev Neurosci 4:901-909. CrossRef Medline
Sethupathy P, Megraw M, Hatzigeorgiou AG (2006) A guide through present computational approaches for the identification of mammalian microRNA targets. Nat Methods 3:881-886. CrossRef Medline

Sim SE, Lee HR, Kim JI, Choi SL, Bakes J, Jang DJ, Lee K, Han K, Kim E, Kaang BK (2013) Elevated RalA activity in the hippocampus of PI3Kgamma knock-out mice lacking NMDAR-dependent long-term depression. BMB Rep 46:103-106. CrossRef Medline

Soriano P (1999) Generalized lacZ expression with the ROSA26 Cre reporter strain. Nat Genet 21:70-71. CrossRef Medline

Sousa-Ferreira L, Garrido M, Nascimento-Ferreira I, Nobrega C, SantosCarvalho A, Alvaro AR, Rosmaninho-Salgado J, Kaster M, Kügler S, de Almeida LP, Cavadas C (2011) Moderate long-term modulation of neuropeptide $\mathrm{Y}$ in hypothalamic arcuate nucleus induces energy balance alterations in adult rats. PLoS One 6:e22333. CrossRef Medline

Stoyanov B, Volinia S, Hanck T, Rubio I, Loubtchenkov M, Malek D, Stoyanova S, Vanhaesebroeck B, Dhand R, Nürnberg B (1995) Cloning and characterization of a $\mathrm{G}$ protein-activated human phosphoinositide-3 kinase. Science 269:690-693. CrossRef Medline

Trajkovski M, Hausser J, Soutschek J, Bhat B, Akin A, Zavolan M, Heim MH, Stoffel M (2011) MicroRNAs 103 and 107 regulate insulin sensitivity. Nature 474:649-653. CrossRef Medline

Villanueva EC, Münzberg H, Cota D, Leshan RL, Kopp K, Ishida-Takahashi R, Jones JC, Fingar DC, Seeley RJ, Myers MG Jr (2009) Complex regulation of mammalian target of rapamycin complex 1 in the basomedial hypothalamus by leptin and nutritional status. Endocrinology 150:45414551. CrossRef Medline

Wu Q, Palmiter RD (2011) GABAergic signaling by AgRP neurons prevents anorexia via a melanocortin-independent mechanism. Eur J Pharmacol 660:21-27. CrossRef Medline

Wu Q, Clark MS, Palmiter RD (2012) Deciphering a neuronal circuit that mediates appetite. Nature 483:594-597. CrossRef Medline

Xu Y, Elmquist JK, Fukuda M (2011) Central nervous control of energy and glucose balance: focus on the central melanocortin system. Ann N Y Acad Sci 1243:1-14. CrossRef Medline

Yang SB, Tien AC, Boddupalli G, Xu AW, Jan YN, Jan LY (2012) Rapamycin ameliorates age-dependent obesity associated with increased mTOR signaling in hypothalamic POMC neurons. Neuron 75:425-436. CrossRef Medline

Zoncu R, Efeyan A, Sabatini DM (2011) mTOR: from growth signal integration to cancer, diabetes and ageing. Nat Rev Mol Cell Biol 12:21-35. CrossRef Medline

Zovoilis A, Agbemenyah HY, Agis-Balboa RC, Stilling RM, Edbauer D, Rao P, Farinelli L, Delalle I, Schmitt A, Falkai P, Bahari-Javan S, Burkhardt S, Sananbenesi F, Fischer A (2011) MicroRNA-34c is a novel target to treat dementias. EMBO J 30:4299-4308. CrossRef Medline 